\title{
What determines the direction of microsaccades?
}

\author{
Frouke Hermens \\ (1) Department of Psychology \\ Royal Holloway, University of London, UK \\ (2) Laboratory of Experimental Psychology \\ University of Leuven, Belgium
}

\author{
Robin Walker \\ Department of Psychology \\ Royal Holloway, University of London, UK
}

During visual fixation, our eyes are not entirely still. Instead, small eye movements,
such as microsaccades, can be observed. We here investigate what determines the
direction and frequency of these microsaccades, as this information might help to
clarify what purpose they serve. The relative contribution of three possible factors was
examined: (1) the orienting of covert attention, (2) the spatial distribution of possible
target locations, and (3) whether monocular or binocular microsaccades are consid-
ered. The orienting of covert attention and the distribution of possible target locations
had a relatively weak effect on microsaccade rates and directions. In contrast, the
classification of microsaccades as binocular (occurring in both eyes simultaneously)
or monocular (observed in one eye only) strongly affected both the rate and the direc-
tion of microsaccades. The results are discussed in the context of existing findings.

Keywords: eye movements, microsaccades, attention

\section{Introduction}

In our day to day life we constantly make eye movements to bring objects of interest onto the fovea. These fast eye movements that bring the eye from one object to another are alternated with periods of visual fixation, in which the eye remains relatively still. However, even during visual fixation, the eye makes small movements. One of these fixational eye movements has properties similar to those of the large saccadic eye movements that shift eye gaze from one object to another. These fixational saccades are known as microsaccades (Kowler \& Steinman, 1980; MartinezConde, 2006; Martinez-Conde, Macknik, Troncoso, \& Hubel, 2009; Rolfs, 2009; Steinman, Haddad, Skavenski, \& Wyman, 1973).

The purpose of these microsaccades has been highly debated (for recent reviews, see Martinez-Conde et al., 2009; Rolfs, 2009). It has been suggested that microsaccades prevent the fading of the retinal image (e.g., Martinez-Conde, Macknik, Troncoso, \& Dyar, 2006) and improve spatial accuracy (Rucci, Iovin, Poletti, \&

Dr. Frouke Hermens, Department of Psychology, Royal Holloway, University of London, Egham, Surrey, UK, TW20 OEX and Laboratory of Experimental Psychology, University of Leuven, Leuven, Belgium, B-3000. This work was supported by ESRC grant RES-000-22-2932. FH is a Postdoctoral Fellow of the Research Foundation-Flanders (FWO-Vlaanderen) and member of a research group supported by a Methusalem grant by the Flemish Government (METH/08/02)
Santini, 2007). Others, however, have argued that other eye movements that occur during visual fixation, such as drift and tremor, in combination with movements of the head, suffice for this purpose (e.g., Collewijn \& Kowler, 2008). Other possible purposes of microsaccades are to bring the eye back to fixation and to realign the two eyes after the occurrence of involuntary slow drift movements (Engbert, 2006; Engbert \& Mergenthaler, 2006).

\section{Microsaccades and attention}

Recent studies have shown that the direction and frequency of microsaccades can be influenced by the orienting of covert attention (Engbert \& Kliegl, 2003a; Hafed \& Clark, 2002; Galfano, Betta, \& Turatto, 2004; Laubrock, Engbert, \& Kliegl, 2005; Laubrock, Engbert, Rolfs, \& Kliegl, 2007; Rolfs, Engbert, \& Kliegl, 2004, 2005; Rolfs, Laubrock, \& Kliegl, 2006). Normally, when we attend to an object, our eyes shift gaze to the attended object (overt shifts of attention). However, it is also possible to attend to an object without shifting gaze (covert attention). The direction of covert attention is often established using a cuing task (Posner, 1980), in which a cue, presented at fixation (endogenous cueing) or in the periphery (exogenous cueing), speeds up responses to a peripheral target if the cue and target direction are congruent. Response times, however, provide only an indirect measure and therefore a more direct measure, that could uncover the direction of covert attention on a trial by trial basis, would be useful (Horowitz, Fine, Fencsik, Yurgenson, $\&$ Wolfe, 2007). Such a measure could exist in the form 
Journal of Eye Movement Research

$3(4): 1,1-20$

of the distribution of microsaccade directions during visual fixation (Engbert \& Kliegl, 2003a; Hafed \& Clark, 2002).

Several studies have shown that microsaccades follow the direction of covert attention (with either a bias towards the direction of endogenously cued attention or away from exogenous shifts of attention; Engbert \& Kliegl, 2003a; Hafed \& Clark, 2002; Galfano et al., 2004; Laubrock et al., 2005, 2007; Rolfs et al., 2004, 2005, 2006; Turatto, Valsecchi, Tame, \& Betta, 2007). However, other studies have failed to find a reliable link between microsaccade direction bias and covert attention (Horowitz et al., 2007; Tse, Sheinberg, \& Logothetis, 2002, 2004; Valsecchi, Betta, \& Turatto, 2007). Several factors could be involved in this discrepancy. For example, studies differed in whether recordings were made from one eye only (monocular recordings; Betta, Galfano, \& Turatto, 2007; Hafed \& Clark, 2002; Galfano et al., 2004) or whether only eye movements that occurred in both eyes simultaneously were considered (binocular microsaccades; Engbert \& Kliegl, 2003a; Rolfs et al., 2004, 2005, 2006; Turatto et al., 2007). Binocular microsaccades have often been found in the horizontal direction (Laubrock et al., 2005; Valsecchi et al., 2007). In contrast, monocularly recorded microsaccades have been reported in other directions as well (Engbert \& Kliegl, 2003b; Engbert, 2006; Hafed, Goffart, \& Krauzlis, 2009; Kloke, Jaschinski, \& Jainta, 2009). Similarly, the spatial distribution of the locations to which covert attention was directed, varied across studies. Either only horizontal directions were cued (Betta et al., 2007; Galfano et al., 2004; Engbert \& Kliegl, 2003a; Valsecchi et al., 2007) or cues could appear in any direction (Hafed \& Clark, 2002; Hafed et al., 2009; Turatto et al., 2007). The distribution of microsaccades directions could therefore be influenced by the distribution of the stimuli across the display, or expectations about where the target could appear (Engbert \& Kliegl, 2003a; Hafed \& Clark, 2002; Galfano et al., 2004; Laubrock et al., 2005, 2007; Rolfs et al., 2004, 2005, 2006; Turatto et al., 2007). However, other studies have failed to find a reliable link between microsaccade direction bias and covert attention (Horowitz et al., 2007; Tse et al., 2002, 2004; Valsecchi et al., 2007). Several factors could be involved in this discrepancy. For example, studies differed in whether recordings were made from one eye only (monocular recordings; Betta et al., 2007; Hafed \& Clark, 2002; Galfano et al., 2004) or whether only eye movements that occurred in both eyes simultaneously were considered (binocular microsaccades; Engbert \& Kliegl, 2003a; Rolfs et al., 2004, 2005, 2006; Turatto et al., 2007). Binocular microsaccades have often been found in the horizontal direction (Laubrock et al., 2005; Valsecchi et al., 2007). In contrast, monocularly recorded microsaccades have been reported in other directions as well (Engbert \& Kliegl, 2003b; Engbert, 2006; Hafed et al., 2009; Kloke et al., 2009). Similarly, the spatial distribution of the locations to which
Hermens, F. \& Walker, R. (2010)

What determines the direction of microsaccades?

covert attention was directed, varied across studies. Either only horizontal directions were cued (Betta et al., 2007; Galfano et al., 2004; Engbert \& Kliegl, 2003a; Valsecchi et al., 2007) or cues could appear in any direction (Hafed \& Clark, 2002; Hafed et al., 2009; Turatto et al., 2007). The distribution of microsaccades directions could therefore be influenced by the distribution of the stimuli across the display, or expectations about where the target could appear.

\section{Microsaccade direction}

The current study was motivated by an apparent discrepancy in the earlier findings. On the one hand, there are indications that microsacccades can be directed in many directions (Hafed et al., 2009; Horwitz \& Albright, 2003) and that they follow cues in many different directions (Hafed \& Clark, 2002; Pastukhov \& Braun, 2010; Turatto et al., 2007). The large extent of possible microsaccade directions is supported by single cell recordings in monkeys demonstrating that cells in the rostral pole of the superior colliculus, involved in the generation of microsaccades, have a wide range of preferred directions (Hafed et al., 2009). On the other hand, binocular microsaccades have mainly been reported in the horizontal direction (Engbert \& Kliegl, 2003a; Laubrock et al., 2005), whereas monocular microsaccades appear to be biased in the vertical direction (Engbert \& Kliegl, 2003b; Engbert, 2006).

There are a number of possible explanations for this apparent discrepancy. In the study by Turatto and colleagues (2007), for example, the data were presented by aligning all microsaccades to one of the possible target locations. It is possible, therefore, that the reported bias occurred in the horizontal direction only and no bias occurred in the other directions. The average histogram would still show a bias towards the target, originating from the bias for the horizontal direction. Similarly, Pastukhov and Braun (2010) investigated the proportion of microsaccades made in the upward and downward directions and found a vertical bias of microsaccades in the direction of the cue. However, by plotting the proportion of microsaccades with a vertical component in the direction of the cue, the possibility remains that microsaccades were mainly biased in the horizontal direction, but the small component in the vertical direction followed the direction of the cue.

Another possibility that would reconcile the contradicting findings would be that the range of cued directions determines the direction of microsaccades. In such an explanation, if cued directions only occur in the horizontal direction, only horizontal microsaccades are found. If cues appear in many different directions, microsaccades will show a wider range of directions.

A third possibility would be that differences in results are the consequence of studies differing in the number (one or two) eyes tracked to measure microsac- 
Journal of Eye Movement Research

$3(4): 1,1-20$

cades. In the study by Hafed and colleagues (2009), for example, microsaccades were recorded in one eye only (monocular recording). Because monocular microsaccades appear to occur in more directions than binocular microsaccades (Engbert \& Kliegl, 2003b; Engbert, 2006), this could explain why a large range of preferred microsaccade directions was found.

Data from Tse et al. (2004) might suggest that microsaccades are consistently biased in the horizontal direction, even when a broad range of stimulus directions and monocular recording is used. By reanalyzing their data from an earlier study (Tse et al., 2002), using an algorithm developed by Engbert and colleagues (Engbert \& Kliegl, 2003a; Engbert \& Mergenthaler, 2006), the monocularly measured microsaccades were found to be biased in the horizontal direction even while cues were appearing in four possible directions. Moreover, no clear indication of a bias in the direction of covert attention was found. The experimental procedure, however, differed substantially from that used by studies showing a relation between the direction of covert attention and microsaccades. The cues were uninformative with respect to the target location, which was varied across 149 positions in the display. Moreover, participants were required to maintain fixation within an distance of $1.5 \mathrm{deg}$ of visual angle from the fixation point (otherwise the trial was aborted and repeated), which could have influenced the occurrence of microsaccades (Poletti \& Rucci, 2010). Furthermore, the paradigm used a sequence of several display changes within a short interval, and it is known that stimulus onsets and changes influence the frequency of microsaccades (Engbert \& Kliegl, 2003a). The above factors could have contributed to why no link between the peripheral cues and microsaccades were found in this particular study (see also Rolfs et al., 2004).

\section{Attentional cueing in multiple directions}

The present study used a covert attention paradigm to examine the link between microsaccade direction and covert attention, using a similar cueing task, identical eye tracking equipment, and the same algorithm to detect microsaccades as previously used (e.g., Engbert \& Kliegl, 2003a). Staying close to the paradigm from earlier studies is important, as the analysis of microsaccades involves the detection of relatively infrequent eye movements (rates of about 1 per second have been consistently reported; Martinez-Conde et al., 2009) with amplitudes close to the intrinsic noise levels of the recording equipment. If any differences are found with respect to the original studies, this can then only be attributed to experimental variations and not to how the data were recorded or analyzed.

The study differed with respect to earlier investigations in that both the position and the number of the possible target locations was varied, not only across horizontal, but also across vertical directions. In one third
Hermens, F. \& Walker, R. (2010)

What determines the direction of microsaccades?

of the trials, targets could appear to the left or right of fixation, indicated by two place-holders positioned along the horizontal axis. In another third of the trials, targets could appear above or below fixation, inside one of the place-holders placed along the vertical axis. In the remaining third of the trials, four possible target locations were used: left of, right of, above, and below fixation. Before the appearance of the target, a centrally presented arrow cued the likely position of the target with an $80 \%$ cue validity. Eye movements were recorded with a video-based eye tracker, resulting in both monocular (occurring in one eye only) and binocular microsaccades (simultaneously occurring in both eyes; Engbert \& Kliegl, 2003b). Three hypotheses were compared: (1) microsaccades follow the direction of covert attention, (2) microsaccade direction is determined by the spatial distribution of the possible target locations, independent of attention, and (3) microsaccade directions depend on whether binocular or monocular microsaccades are examined. If microsaccades follow attention, a bias in the direction of the arrow cue is expected after cue onset, but not before. If microsaccades are related to the possible target locations, independent of attention, no such bias in the direction of the arrow should be present after cue onset. Instead, both before and after cue onset, horizontal place-holders should induce a horizontal bias, and vertical place-holders a vertical bias. Finally, if microsaccade directions are determined by whether the criteria for detection require the microsaccade to occur in both eyes simultaneously ('binocular microsaccades') or not ('monocular microsaccades'), binocular microsaccades shoud be biased horizontally, while monocular microsaccades are expected to be in both the horizontal and vertical directions, independent of the direction of the cue, or the placement of the place-holders indicating the possible saccade target directions (Engbert \& Kliegl, 2003b).

\section{Methods}

\section{Participants}

Eight participants took part in the experiment. Two of the participants were the authors, while the other six participants were undergraduate students at Royal Holloway, University of London. All gave their informed consent for their participation in the experiment. The students were paid $£ 10$ for their participation. The experimental procedure was approved by the local ethics committee.

\section{Apparatus}

A 21 inch CRT screen presented the stimuli at a refresh rate of $100 \mathrm{~Hz}$. Stimulus presentation was controlled by an AMD Athlon 2400 PC. A second, Pentium 4 PC was used to record the eye movement 
Journal of Eye Movement Research

$3(4): 1,1-20$

data. Eye movements were measured using the Eyelink II video-based eye tracker (SR Research Osgood, ON, Canada), which was mounted on a head-and-chin rest positioned at a distance of $57 \mathrm{~cm}$ from the computer screen. Horizontal and vertical eye positions for both eyes were sampled at a rate of $500 \mathrm{~Hz}$ (pupil-only mode).

\section{Stimuli}

Figure 1a shows the stimulus sequence. A fixation symbol was presented in the center of the screen surrounded by two or four place-holder circles. The fixation symbol measured $1 \mathrm{~cm}$ by $1 \mathrm{~cm}$ ( 1 by 1 degree of visual angle) and the circles were $0.7 \mathrm{~cm}(0.7$ degrees $)$ in diameter. The circles were positioned at a distance of $9 \mathrm{~cm}$ (9 degrees) from the center of the screen. Following the fixation interval, the cue was presented by the removal of two lines from the fixation symbol, turning the diamond shape into an arrow. After a random period of $1500 \mathrm{~ms}$ to $2000 \mathrm{~ms}$, the target, a star-shape measuring $0.7 \mathrm{~cm}$ by $0.7 \mathrm{~cm}$ ( 0.7 by 0.7 degrees) appeared inside one the place-holders. After the participants made a saccade, the stimuli were removed from the screen and feedback on the saccadic reaction time was provided in the center of the screen. This feedback consisted of the text ' $\mathrm{RT}={ }^{\prime}$ followed by a number indicating the response time in milliseconds and served to keep participants motivated across the experiment to produce fast response times. A blank screen for $500 \mathrm{~ms}$ served as the inter-trial interval. All stimuli were presented in white on a black background.

Figure $1 \mathrm{~b}$ illustrates the three possible place-holder and target positions. Place-holders and targets could appear on the horizontal axis ('horizontal'), on the vertical axis ('vertical'), or on both the horizontal and vertical axes ('horizontal and vertical').

\section{Design}

The experiment was run in blocks of 60 trials. The number and position of the possible target locations was varied between blocks, counter-balanced for each participant, and their order randomized across participants. The direction of the arrow and the target was varied randomly across trials, with the restrictions that each arrow direction occurred equally often within a block of trials and that cue validity was $80 \%$, meaning that the target appeared inside the place-holder that was pointed at on $80 \%$ of the trials and in one of the other place-holders on the remaining $20 \%$ of the trials (not necesarily the place-holder opposite to the cue direction in the combined horizontal and vertical cue condition). Participants performed each condition (horizontal, vertical, or horizontal+vertical) either 240 times (authors FH and RW) or 120 times (the remaining participants), which took 2 hours or 1 hour respectively to complete. The experiment was split in sessions of
Hermens, F. \& Walker, R. (2010)

What determines the direction of microsaccades? a) Stimulus sequence

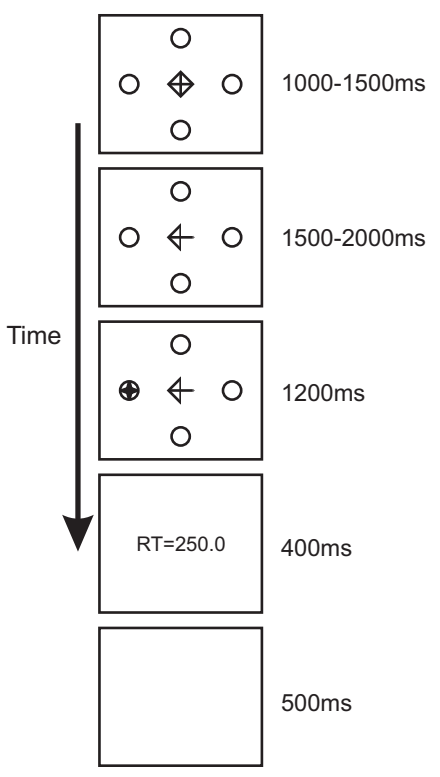

b) Possible target locations
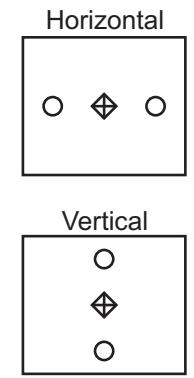

Horizontal and vertical

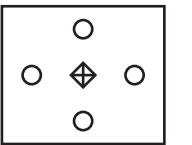

Figure 1. a) Stimulus sequence. A fixation symbol was presented together with two or four place-holder circles for a duration between $1000 \mathrm{~ms}$ and $1500 \mathrm{~ms}$, after which two of the lines of the fixation symbol were removed, turning it into an arrow. After a delay of between $1500 \mathrm{~ms}$ and $2000 \mathrm{~ms}$, the target (a star-shape) was presented inside one of the placeholders. Once a saccade was detected after target onset, the stimuli were removed from the screen and feedback on the reaction time was provided for $400 \mathrm{~ms}$. A blank screen presented for $500 \mathrm{~ms}$ served as the inter-trial interval. The stimuli are illustrated in reverse contrast (in the experiment stimuli were presented in white on a dark background). b) Illustration of the possible target locations, indicated by the locations of the place-holders. Either targets appeared along the horizontal axis, along the vertical axis, or along the horizontal and vertical axis.

half an hour each, separated by intervals of at least 2 hours.

\section{Procedure}

Participants were seated with their head restrained by a head-and-chin rest. A calibration procedure was performed before the experiment and each new block of trials, during which ten small targets were presented on a three by three grid in a random order, with the first and last target presented in the center of the screen. Participants were asked to fixate each of the targets. Once all recorded fixations were aligned on a three by three grid and the recording of the first and last fixation overlapped sufficiently, the calibration setting was accepted.

Calibration was followed by a drift correction in which participants were asked to fixate a centrally presented dot and press the spacebar of the computer key- 
Journal of Eye Movement Research $3(4): 1,1-20$

board in front of them. This drift correction was repeated after each tenth trial. Participants performed at least 20 practice trials before the experiment.

On each trial, a central fixation symbol was presented for a duration between $1000 \mathrm{~ms}$ and $1500 \mathrm{~ms}$, followed by the arrow cue for $1500 \mathrm{~ms}$ to $2000 \mathrm{~ms}$, after which the target appeared inside one of the placeholders. Participants were asked to fixate the fixation symbol and the arrow at the intersection point of the horizontal and vertical line and to shift gaze to the target as soon as it appeared. They were informed that on $80 \%$ of the trials, the target would appear inside the place-holder that the arrow pointed at and in the remaining $20 \%$ of the trials inside (one of) the other place-holder(s), and that they should try and respond as quickly as possible to the targets, trying to avoid making a saccade in the wrong direction. Response time feedback was provided after each trial, based on the time from target onset until moment that the velocity and acceleration of the cyclopedian eye exceeded a set criterion ( $80 \mathrm{deg} / \mathrm{sec}$ and 3,000 deg $/ \mathrm{s}^{2}$ respectively).

\section{Data analysis}

Trials where a saccade was initiated too quickly (less than $80 \mathrm{~ms}$ after target onset) or too slowly (more than $600 \mathrm{~ms}$ after target onset) as well as saccades in the wrong direction (more than 2 degrees away from the target) and saccades that contained a blink were removed from the analysis. These criteria led to the exclusion of $3.8 \%$ of the trials of the condition with horizontal targets, $12.6 \%$ of the condition with vertical targets, and $12.7 \%$ of condition the horizontal and vertical targets.

Microsaccades were detected using the algorithm of Engbert and colleagues (Engbert \& Kliegl, 2003a; Engbert \& Mergenthaler, 2006). Sections of the eye trace during which the velocity exceeded a threshold of six times the standard deviation (computed separately for horizontal and vertical directions) and that lasted more than 3 samples $(6 \mathrm{~ms})$ were classified as microsaccades if the amplitude did not exceed 30 pixels (1.1 degrees; the same criterion as used by Hermens, Zanker, \& Walker, 2010). Examples of eye traces illustrating this procedure can be found in previous publications (e.g., Engbert \& Kliegl, 2003a; Engbert, 2006; Hermens et al., 2010). Binocular microsaccades were defined as those eye movements that occurred in both eyes at the same time (i.e., at least one sample overlapped in time). Monocular microsaccades fell within two categories: Eye movements that occurred in one eye, irrespective of whether a microsaccade also occurred in the other eye ('non-unique monocular microsaccades') and eye movements that occurred in uniquely one eye ('uniquely monocular microsaccades').

The amplitude and direction of binocular microsaccades was determined by using the average horizontal and vertical displacement across the two eyes (Engbert
Hermens, F. \& Walker, R. (2010)

What determines the direction of microsaccades?

\& Kliegl, 2003a; Engbert \& Mergenthaler, 2006). This was possible, because the amplitude and direction of microsaccades in the two eyes were highly correlated (amplitude: $\mathrm{r}=0.95, \mathrm{p}<0.01$; direction: $\mathrm{r}=0.97, \mathrm{p}<0.01$, across all 13637 binocular microsaccades in the present study).

To plot the microsaccade rate as a function of time, a window of $100 \mathrm{~ms}$ was shifted along the time axis in steps of $1 \mathrm{~ms}$, counting the average number of microsaccades (per second) that fell inside the window. The temporal onset and offset of the microsaccades were aligned to the onset of the cue.

\section{Results}

\section{Response times}

Response times (RTs) were shorter for valid cues than for invalid cues (Figure 2; main effect of cue validity: $F(1,7)=20.14, p=0.003$, main effect of target location: $F(2,14)=8.12, p=0.005$, interaction: $F(2,14)=9.45$, $\mathrm{p}=0.003)$. Posthoc tests revealed significant congruency effects for horizontal targets $(t(7)=4.46, p=0.003)$, vertical targets $(\mathrm{t}(7)=3.65, \mathrm{p}=0.008)$ and in the condition combining horizontal and vertical targets $(t(7)=4.27$, $\mathrm{p}=0.04)$. Within the combined horizontal and vertical cue and target condition, faster RTs were obtained both on horizontal $(\mathrm{t}(7)=6.01, \mathrm{p}=0.001)$ and vertical cue trials $(\mathrm{t}(7)=2.81, \mathrm{p}=0.026)$.

\section{Microsaccade frequency}

Table 1 shows the average binocular and monocular microsaccade rates (number of microsaccades per second) across the $2500 \mathrm{~ms}$ of fixation (from $-1000 \mathrm{~ms}$ before cue onset to $1500 \mathrm{~ms}$ after cue onset) for each participant in the experiment. Monocular rates in this table refer to eye movements occurring in one eye uniquely (nonunique monocular rates can be obtained by adding the rates for the reported binocular and uniquely monocular microsaccades).

In agreement with earlier findings (e.g., Engbert \& Kliegl, 2003a), the present study revealed substantial variations in microsaccade rates across participants. For example, for binocular microsaccades in the horizontal targets condition, the lowest rate was $0.28 \mathrm{mi}-$ crosaccades per second and the highest 1.82, showing a 6.5-fold difference. Despite this variability, microsaccade rates appear to be similar across conditions. For monocular microsaccades slightly higher rates were found for the vertical targets and for microsaccades in the right eye (although both effects failed to reach significance in a repeated measures ANOVA; effect of target location: $\mathrm{F}(2,14)=1.74, \mathrm{p}=0.21$, effect of eye: $F(1,7)=1.67, p=0.24$, interaction: $F(2,14)=0.95$, $\mathrm{p}=0.41$ ). A comparison between monocular (summing the rates for each of the two eyes) and binocular microsaccade rates revealed significantly higher rates of binocular microsaccades $(F(1,7)=5.59, p=0.50$; 


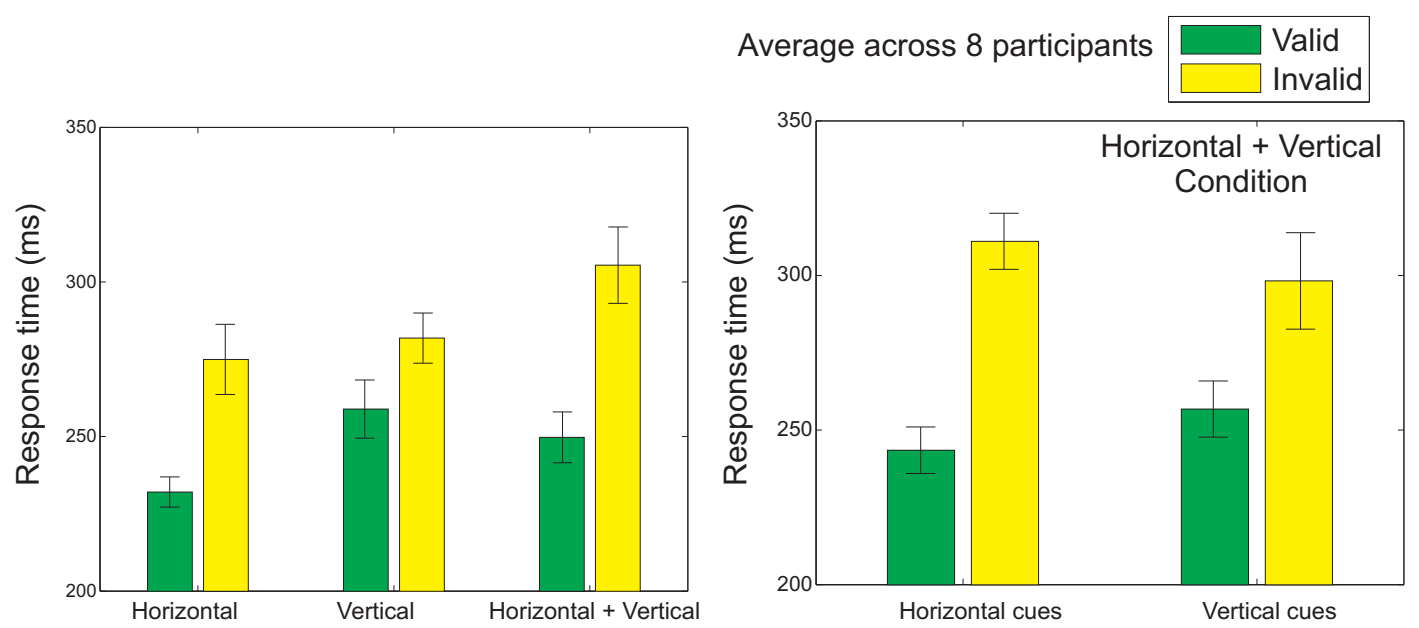

Figure 2. Left: Average saccadic response time to the target after the presentation of a valid (green bars) or an invalid arrow cue (yellow bars), for the three different conditions (the horizontal and vertical conditions, where two possible target locations were used, and the combined horizontal and vertical condition, with four possible target locations). Right: Average response saccadic latencies for the combined horizontal and vertical condition, for the two different main cue directions (horizontal and vertical). The error bars show the standard error of the mean across participants.

Table 1

The average microsaccade frequency across the $2500 \mathrm{~ms}$ interval around cue onset for each of the participants (numbered 1-8, 'Su' column). The columns labeled 'BinH', 'BinV' and 'BinHV' show the rates for binocular microsaccades for horizontal, vertical, and combined horizontal and vertical targets respectively. The remaining columns show the rates for uniquely monocular microsaccades in the different conditions for the two eyes ( $L=l e f t, R=r i g h t)$.

\begin{tabular}{|c|c|c|c|c|c|c|c|c|c|}
\hline$\overline{\mathrm{Su}}$ & $\mathrm{BinH}$ & $\operatorname{BinV}$ & BinHV & $\begin{array}{l}\text { MonH- } \\
\text { L }\end{array}$ & $\begin{array}{l}\text { MonV- } \\
\text { L }\end{array}$ & $\begin{array}{l}\text { MonHV- } \\
\text { L }\end{array}$ & $\begin{array}{l}\text { MonH- } \\
\text { R }\end{array}$ & $\begin{array}{l}\text { MonV- } \\
\text { R }\end{array}$ & $\begin{array}{l}\text { MonHV- } \\
\text { R }\end{array}$ \\
\hline 1 & 1.22 & 1.45 & 1.40 & 0.40 & 0.37 & 0.46 & 1.00 & 1.29 & 1.32 \\
\hline 2 & 1.45 & 1.71 & 1.45 & 0.60 & 0.73 & 0.72 & 0.66 & 0.64 & 0.84 \\
\hline 3 & 1.02 & 1.12 & 1.39 & 0.76 & 0.76 & 1.03 & 1.22 & 1.52 & 1.24 \\
\hline 4 & 0.30 & 0.27 & 0.25 & 0.47 & 0.46 & 0.37 & 0.68 & 0.81 & 0.57 \\
\hline 5 & 1.82 & 1.81 & 1.56 & 1.03 & 1.06 & 0.90 & 0.56 & 0.68 & 0.65 \\
\hline 6 & 0.44 & 0.74 & 0.72 & 0.59 & 0.42 & 0.51 & 0.42 & 0.34 & 0.50 \\
\hline 7 & 0.28 & 0.50 & 0.32 & 0.18 & 0.46 & 0.46 & 0.23 & 0.39 & 0.22 \\
\hline 8 & 0.89 & 0.94 & 0.81 & 0.30 & 0.25 & 0.19 & 0.60 & 0.64 & 0.52 \\
\hline Mean & 0.93 & 1.07 & 0.99 & 0.54 & 0.56 & 0.58 & 0.67 & 0.79 & 0.73 \\
\hline
\end{tabular}

with a marginally significant effect of target location: $\mathrm{F}(2,14)=3.56, \mathrm{p}=0.056$, and no interaction $\mathrm{F}(2,14)=0.69$, $\mathrm{p}=0.52)$. Unexpectedly, higher binocular microsaccade rates were found for the vertical than for the horizontal target condition $(\mathrm{F}(1,7)=16.63, \mathrm{p}=0.005)$.

To investigate the time-course of the microsaccade rate, the frequency at which microsaccades occurred was plotted as a function of time before (negative values on the horizontal axis) and after (positive values) cue onset. Figure 3 presents these time-dependent rates separately for binocular microsaccades, non-unique monocular microsaccades and uniquely monocular microsaccades. Note that in these plots, the scaling of the vertical axis is different for the different types of microsaccades. For binocular and monocular non-unique microsaccades, the typical pattern (the microsaccade 'signature'; Engbert \& Kliegl, 2003a) can be observed, with a decrease of the rate after stimulus onset, followed by an increase above baseline. The signature is strongly reduced in amplitude if uniquely monocular microsaccades are considered. For binocular and nonunique monocular microsaccades, a decreasing microsaccade rate before the onset of the cue and before the onset of the target is found. The histograms for binocular microsaccades show that the higher rate for the vertical targets arises between $-600 \mathrm{~ms}$ to $-400 \mathrm{~ms}$ before cue onset $(\mathrm{F}(2,14)=4.06, \mathrm{p}=0.041)$, which appears to be mainly the result of a difference in the microsaccades rate in the right eye. 

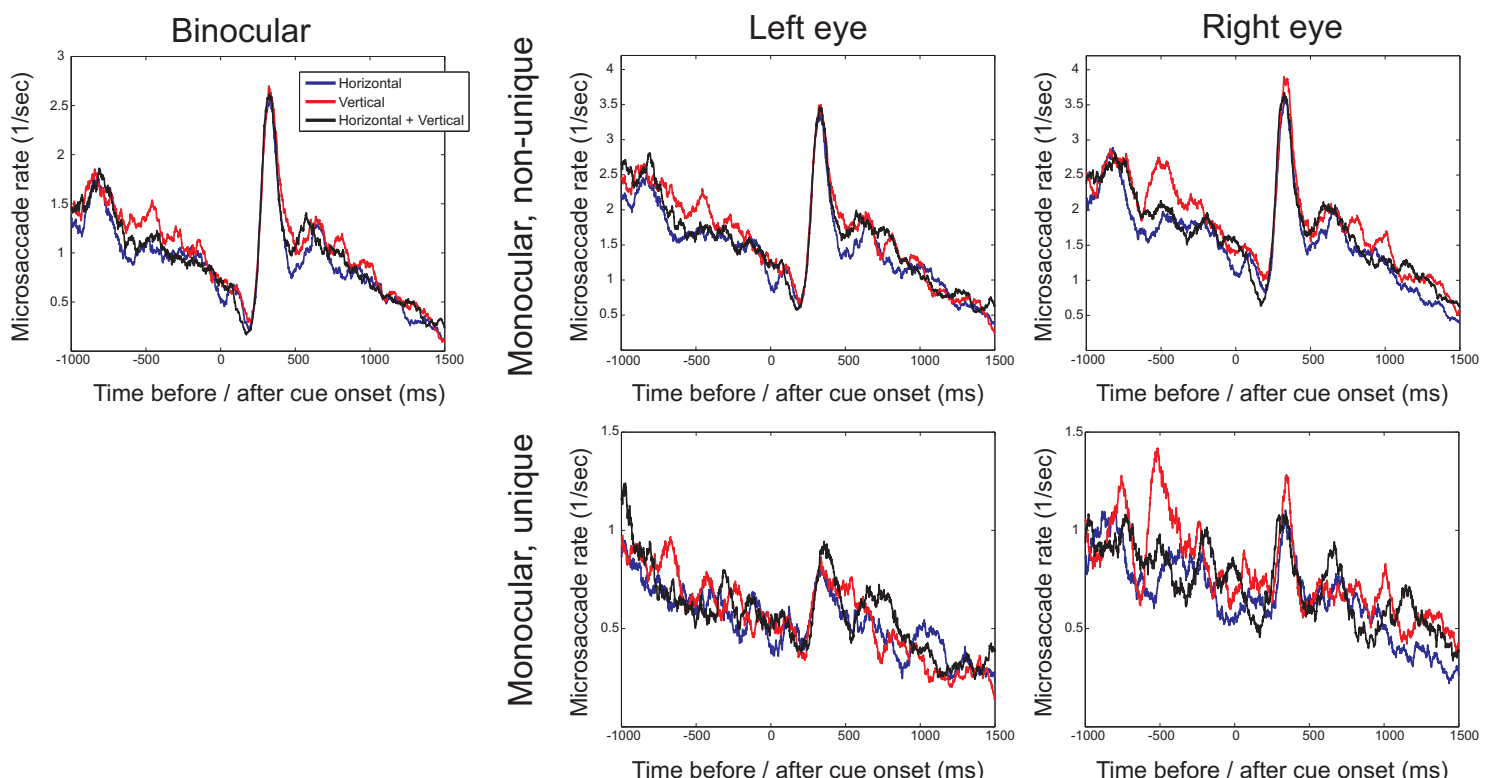

Figure 3. The microsaccade rate (the number of microsaccades per second) as a function of the time before (negative values) and after (positive values) cue onset for the three different directions in which cues occurred (horizontal, vertical, horizontal and vertical). Separate plots show binocular microsaccades (left) and monocular microsaccades (right). Monocular rates are either from non-unique monocular microsaccades (with the possibility that a microsaccade occurring simultaneously in the other eye, comparable to when only eye would have been monitored) or from uniquely monocular microsaccades (microsaccades in one eye, but not the other). Note that the scaling on the vertical axis differs for the different types of microsaccades.

\section{Microsaccade direction}

The role of covert attention. Figures 4 and 5 show polar plots of the distribution of microsaccade directions before and after cue onset, respectively, for the different conditions. A comparison between the distributions of microsaccade directions before and after cue onset does not reveal any clear differences. Moreover, after cue onset, no clear bias in the direction of the cue seems to be present. If microsaccades would have been strongly biased in the direction of attention, its distribution would have been expected to align with the vertical axis for vertically oriented cues. Although a slight shift towards more vertical directions might be observed for the vertical cue condition, it is clearly not the case that the entire distribution has shifted towards the vertical. Moreover, the slight shift towards the vertical for vertical cues appears to occur already before cue onset, suggesting a role for the distribution of possible target locations, in addition to the effects of attention. Such observations were confirmed by a statistical analysis. For example, for binocular microsaccades after cue onset, no significant differences in the proportions of leftward and rightward microsaccades were found for leftward and rightward cues (interaction between cue direction and microsaccade direction: $\mathrm{F}(1,7)=0.28, \mathrm{p}=0.62$, main effect of cue direction: $F(1,7)=0.44, p=0.53)$. Likewise, no significant differences were found in the proportion of upward and downward microsaccades for upward and downward cues (interaction effect: $F(1,7)=2.11, p=0.19$, main effect of cue direction: $F(1,7)=1.28, p=0.30)$. More detailed analyses will be presented when the time-course of the directional microsaccade rates is investigated (later in this results section).

The distribution of possible target locations. Visual inspection of the histograms of microsaccade direction for the binocular microsaccades before cue onset (Figure 4) suggests that horizontal targets lead to horizontal microsaccade directions, whereas the condition with vertical targets and the combined horizontal and vertical target direction condition result in a slightly larger bias of microsaccade directions towards the vertical axis. This difference appears to be present before and after cue onset, suggesting a role of the distribution of the place-holders in the display.

This observation was confirmed by statistical analyses. Across all conditions, slightly more horizontal than vertical binocular microsaccades were found before cue onset $(\mathrm{F}(1,7)=4.35, \mathrm{p}=0.076)$. More importantly, the distribution of horizontal and vertical microsaccades differed across the three possible target distributions (interaction between microsaccade direction and target distribution: $\mathrm{F}(2,14)=4.89, \mathrm{p}=0.025)$. In the condition with the horizontal targets, more horizontal microsaccades were made than in the other two conditions $(\mathrm{F}(1,7)=4.422, \mathrm{p}=0.032$; difference contrast). The horizontal condition also had fewer vertical microsaccades $(F(1,7)=3.77, p=0.049)$. Similar effects were found for the non-unique monocular mi- 


\section{Binocular microsaccades Monocular microsaccades before cue onset before cue onset}
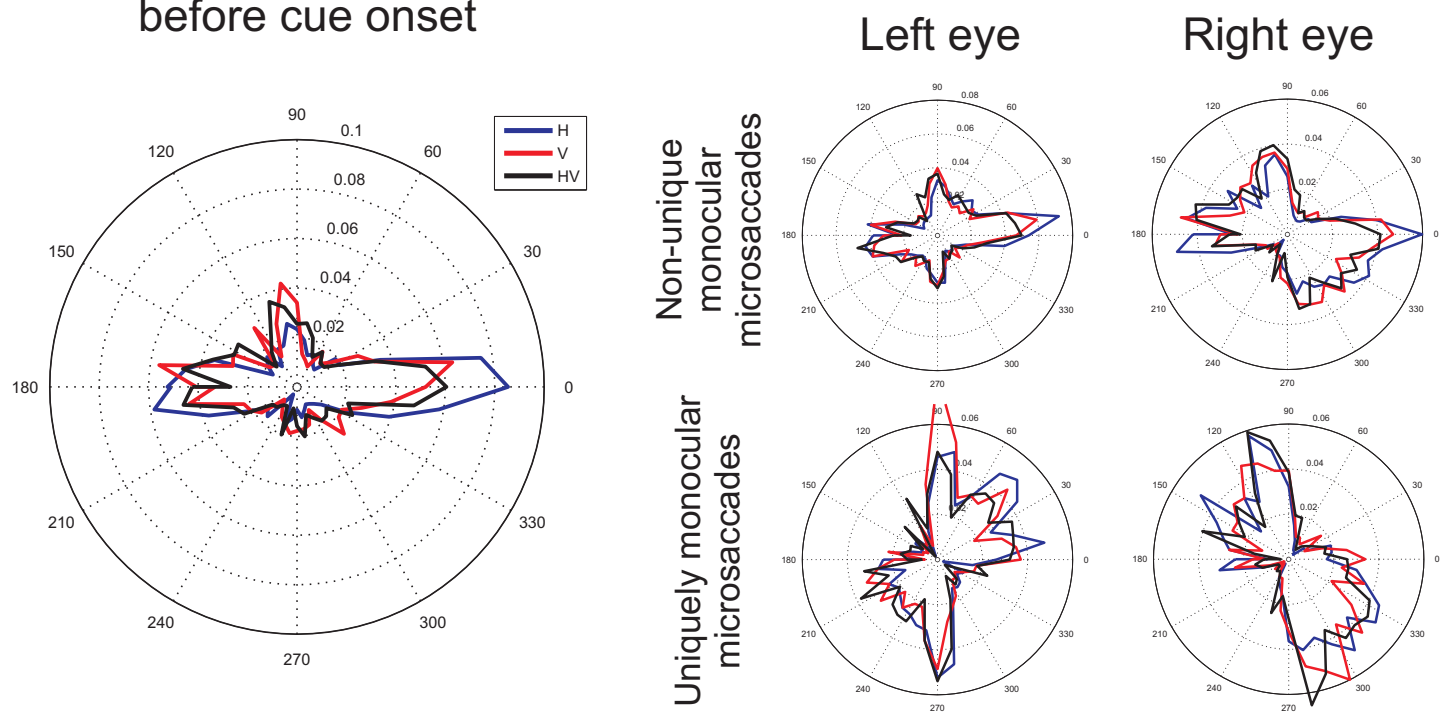

Figure 4. Average histograms (proportions of microsaccades) across participants of the microsaccade directions before cue onset (from $-1000 \mathrm{~ms}$ to $0 \mathrm{~ms}$ before cue onset), during which the fixation symbol was shown to the participant. Blue, red, and black lines show the distribution of microsaccade directions for the horizontal, vertical and combined horizontal and vertical conditions respectively. On the left, histograms of microsaccades that occurred simultaneously in both eyes are shown. On the right, microsaccades from data of one eye only are shown, with in the top two plots, all the microsaccades that occurred in the indicated eye, independent of whether a microsaccade also occurred in the other eye and in the bottom two plots (comparable to when only one eye would have been recorded), the microsaccades that occurred uniquely in the indicated eye.

crosaccades. Overall, however, no difference in the percentage of horizontal and vertical microsaccades were found (left eye: $\mathrm{F}(1,7)=0.88, \mathrm{p}=0.38$; interaction with condition: $F(2,14)=2.22, p=0.15$; right eye: $F(1,7)=1.17$, $\mathrm{p}=0.32$ ). As for the binocular microsaccades, the distribution of non-unique monocular microsaccade directions was significantly influenced by the distribution of possible target locations $(\mathrm{F}(2,14)=5.17, \mathrm{p}=0.021)$. This interaction between the target distribution and microsaccade bias was the consequence of more horizontal microsaccades for horizontal targets $(F(2,14)=5.3$, $\mathrm{p}=0.020$ ). Uniquely monocular microsaccades showed a general bias towards the vertical. This bias was statistically significant for the left eye, where more vertical than horizontal microsaccades were found $(F(1,7)=8.55$, $\mathrm{p}=0.022)$. This bias, however, was independent of the stimulus condition $(\mathrm{F}(2,14)=0.23, \mathrm{p}=0.79)$. Moreover, the vertical bias did not reach statistical significance for the right eye, where horizontal and vertical microsaccade frequencies were not significantly different $(\mathrm{F}(1,7)=4.16, \mathrm{p}=0.081)$. Also for this eye, there was no interaction with the stimulus condition $(\mathrm{F}(2,14)=1.41$, $\mathrm{p}=0.28$ ).

Binocular versus monocular microsaccades. The histograms (Figures 4 and 5) show a clear difference between binocular and monocular microsaccades, replicating the results from Engbert and colleagues (Engbert \& Kliegl, 2003b; Engbert, 2006). Binocular microsac- cades show a horizontal bias, whereas non-unique monocular microsaccades display a combined horizontal and vertical bias. In addition, microsaccades occurring in one eye only were found to be biased in the vertical direction. These observations were confirmed by a statistical analysis. In a 3x2 (possible target locations $\mathrm{x}$ binocular versus monocular) repeated measures ANOVA the proportion of horizontal and vertical microsaccades was compared. Before cue onset, a higher proportion of horizontal microsaccades was found in the binocular than in the non-unique monocular $(\mathrm{F}(1,7)=26.73, \mathrm{p}=0.001)$ and the uniquely monocular microsaccades data $(\mathrm{F}(1,7)=22.14, \mathrm{p}=0.002)$ and these effects varied slightly with the number of possible target locations (interaction effects: $\mathrm{F}(2,14)=2.67$, $\mathrm{p}=0.10$ and $\mathrm{F}(2,14)=3.52, \mathrm{p}=0.058$ respectively). Likewise, fewer vertical binocular microsaccades were found compared to non-unique monocular microsaccades $(\mathrm{F}(1,7)=6.42, \mathrm{p}=0.039)$ and uniquely monocular microsaccades $(\mathrm{F}(1,7)=8.27, \mathrm{p}=0.024)$, independent of the possible target locations $(\mathrm{F}(2,14)=0.82, \mathrm{p}=0.46$ and $\mathrm{F}(2,14)=0.32, \mathrm{p}=0.73$ respectively). A comparison between the non-unique and the uniquely monocular microsaccades showed more horizontal non-unique monocular microsaccades $(\mathrm{F}(1,7)=15.14, \mathrm{p}=0.006)$ and fewer vertical non-unique monocular than uniquely monocular microsaccades $(F(1,7)=8.45, p=0.023)$. 


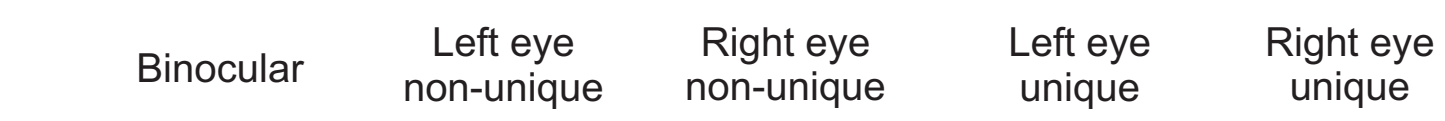
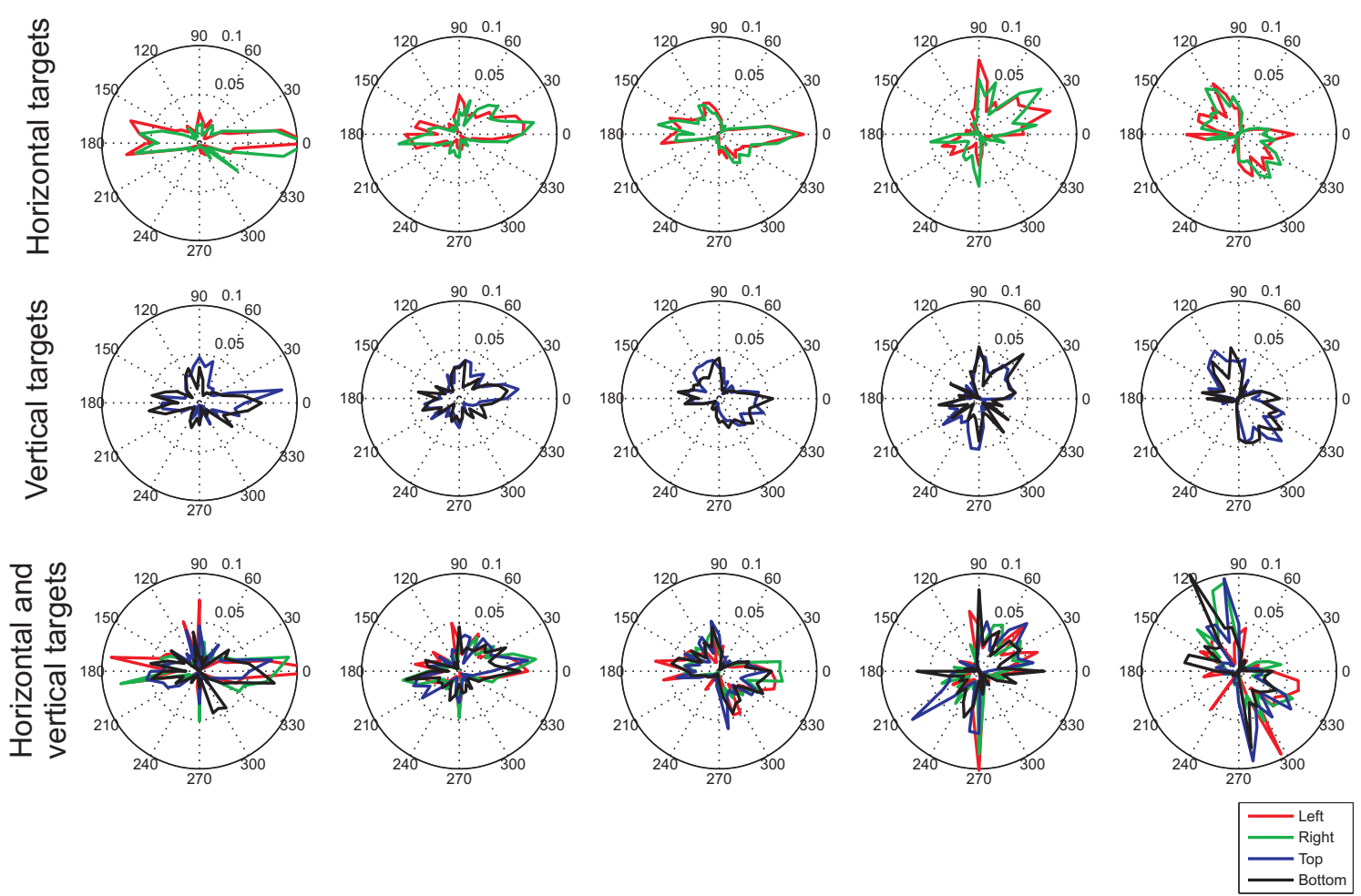

Figure 5. Histograms of microsaccade directions after cue onset (from $0 \mathrm{~ms}$ to $1500 \mathrm{~ms}$ after cue onset), during which participants were presented with the arrow cue. The histograms (of proportions of microsaccades) were constructed by taking the average across the histograms for each participant separately. The different colors show the different cue directions (left, right, up, down). Each of the rows present the histograms for the different target conditions (horizontal, vertical, and combined horizontal and vertical targets). The leftmost column shows binocular microsaccades, the next two columns show histograms for monocular microsaccades that could also occur in the other eye at the same time. The rightmost two columns show the histograms for monocular microsaccades that occurred uniquely in one eye.

\section{Time-course of microsaccade bias}

Previous studies have suggested that a bias of microsaccades in the direction of covert attention only occurs during a particular time interval after cue onset. For example, Engbert and Kliegl (2003a) found that microsaccades follow the direction of an arrow cue mainly between $300 \mathrm{~ms}$ to $400 \mathrm{~ms}$ after cue onset. For color cues, the bias in microsaccade directions was found between $350 \mathrm{~ms}$ and $600 \mathrm{~ms}$ after cue onset. In contrast, peripheral onsets serving as cues were found to first induce a bias towards the direction of the cue (between $20 \mathrm{~ms}$ to $200 \mathrm{~ms}$ after cue onset), followed by a bias away $600 \mathrm{~ms}$ to $800 \mathrm{~ms}$ after cue onset (Laubrock et al., 2005) (for an overview, see Engbert, 2006). Therefore, our earlier analysis, which pooled all microsaccades across a relatively long interval, could have obscured possible microsaccade biases within a smaller temporal interval. Moreover, the relatively large integration interval could have led to biases that occurred in opposite directions within the interval to cancel each other out, with a zero overall bias as a consequence. These pos- sibilities were investigated by plotting microsaccade signatures (microsaccade rates across time) separately for different microsaccade directions. Furthermore, the range of microsaccade directions was divided into four quadrants. Binocular microsaccades with an angular direction between -45 and 45 degrees were classified as rightward microsaccades, those with a direction between 45 and 135 degrees as upward, those with a direction between 135 and 180 and between -135 and -180 degrees as leftward, and those with a direction between -45 and -135 as downward microsaccades. The same method as for the overall microsaccade rates was used to compute the directional microsaccade rate as a function of time (for details, see data analysis section), using a moving window analysis with time-steps of $1 \mathrm{~ms}$ and a window size of $100 \mathrm{~ms}$.

Average rates in each of the cardinal directions for the horizontally (top row) and vertically oriented cues (bottom row) are shown in Figure 6. As in the polar histograms (Figures 4 and 5), more horizontal (red and green curves in Figure 6) than vertical microsaccades (black and blue curves) are found (confirmed by statis- 
tical tests comparing the mean horizontal and vertical microsaccade rates across the entire $2500 \mathrm{~ms}$ interval, for leftward cues: $t(7)=2.79, p=0.027$, and rightward cues: $t(7)=2.52, p=0.040)$. Weak effects of the distribution of possible target locations appear to occur both before and after cue onset, however, failing to reach significance (interaction between place-holder and microsaccade direction, before cue onset: $F(1,7)=3.67$, $p=0.096$; after cue onset: $F(1,7)=0.71, p=0.43$; in the presence of significant main effects of place-holder and microsaccade directions). Interestingly, although the polar histograms did not show any clear effects of attention, the time-course plots suggests that attention does affect the direction of microsaccades between $200 \mathrm{~ms}$ and $450 \mathrm{~ms}$ after cue onset. This interval is in agreement with that proposed by Laubrock and colleagues (in press) and is similar to the interval found by Engbert and Kliegl (2003a) using arrows as cues. During the interval, the frequency of microsaccades in the cued direction is increased with respect to the frequency in other directions, showing a significant bias in the average rate following the direction of the cue (compared to opposite to the cue), both for horizontal $(t(7)=2.36, p=0.050)$ and vertical cues $(t(7)=2.58$, $\mathrm{p}=0.036)$.

Similar effects of the direction of the cue on the timecourse of microsaccade directions were found for the condition that combined horizontal and vertical cues (Figure 7), showing significantly higher rates of microsaccades in the direction of the cue than away from it on vertical cue trials in the $200 \mathrm{~ms}-450 \mathrm{~ms}$ interval $(t(7)=2.97, p=0.020$, two-tailed). For horizontal cues, this increase was significant only in a one-tailed test $(t(7)=1.91, p=0.049$, one-tailed).

\section{Microsaccade amplitude}

Another discrepancy in the literature concerns the typical amplitude of microsaccades (Collewijn \& Kowler, 2008; Martinez-Conde et al., 2009). Generally, earlier studies reported microsaccades amplitudes up to 12 arc minutes (Collewijn \& Kowler, 2008), whereas more recent studies (those using video-based eye trackers) typically use an amplitude criterion of 1 degree of visual angle (60 minutes of arc) to distinguish microsaccades from saccades. To investigate this issue, the range of binocular microsaccade amplitudes in the present study is plotted in Figure 8a, showing that most detected microsaccades had an amplitude smaller than 0.5 degrees (30 minutes of arc). To investigate the consequence of setting a particular amplitude criterion, the main analyses reported so far were repeated with a range of different amplitude thresholds. Figures $8 \mathrm{~b}$ and $8 \mathrm{c}$ show that decreasing the amplitude threshold to 0.5 or 0.25 degrees did not alter the results in a systematic way. The typical microsaccade signature (a decrease in the rate followed by an increase) continued to be found and the direction of microsaccades in the absence of a directional cue remained along the horizontal direction. The bias after cue onset in the direction of the cue, however, appeared to be influenced by restricting the analysis to smaller amplitude microsaccades (Figures $8 \mathrm{~d}$ ), with weaker biases in the direction of the cue for smaller microsaccade amplitudes.

\section{Monocular and binocular microsaccades}

The observation that microsaccades can occur in uniquely one eye is not undisputed. Whereas recent studies (Engbert \& Kliegl, 2003b; Engbert, 2006; Kloke et al., 2009) reported a relatively large proportion of uniquely monocular microsaccades, earlier studies have suggested that microsaccades always occur in both eyes simultaneously (for an overview, see Collewijn \& Kowler, 2008). To investigate whether monocular microsaccades in the present experiment were in any way related to the eye tracker's internal noise, we repeated the experiment with two artificial ('dummy') eyes to establish whether the algorithm (Engbert \& Kliegl, 2003a; Engbert \& Mergenthaler, 2006), in combination with the equipment (Eyelink II), results in any detected microsaccades in the absence of eye movements. Two push-pins (diameter: $5 \mathrm{~mm}$ ) served as the pupils of the dummy eyes. The system was first calibrated using a human subject, after which the dummy pupils took the place of the human observer. If required, the threshold for pupil detection was raised slightly after calibration. Drift correction was performed as in the original experiment, after each 10th trial and before the start of the experiment. The experiment was repeated five times, resulting in data for five different calibrations (of two different human observers). Across the five repetitions, with a total continuous recording time of 25 minutes, 0 binocular microsaccades, 51 left eye and 19 right eye microsaccades were detected. This shows that the number of false detections by the algorithm is low overall and absent when the binocular microsaccade criterion is applied. These results support the notion that microsaccades detected in the main experiment reported here were due to genuine eye movements of the participants rather than noise in the recordings of the Eyelink II.

To further investigate the difference between binocular and monocular microsaccades, Figure 9 examines the relation between the saccade amplitude and peak velocity (black dots) and the histograms of saccade amplitudes (red bars in the background) for the different types of microsaccades. Although the uniquely monocular microsaccades show a bias towards lower saccade amplitudes, they do show an approximately linear relation between saccade amplitude and peak velocity, known as the main sequence (Zuber, Stark, \& Cook, 1965), just like binocular microsaccades.

So far, we have restricted our analysis to microsaccades with a duration of at least $6 \mathrm{~ms}$ (for an overview of minimum microsaccade durations used in previous 


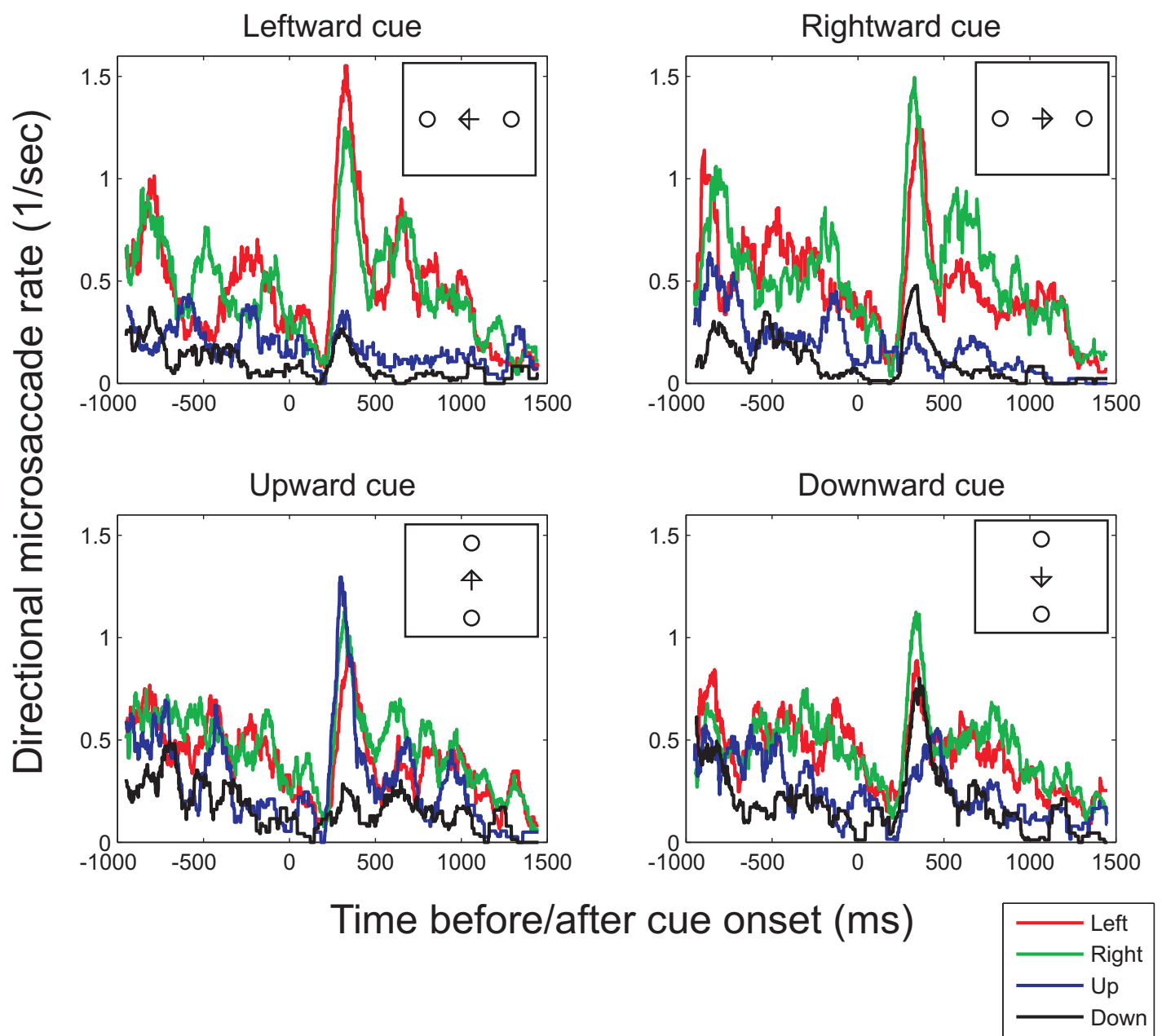

Figure 6. Binocular microsaccade rates in each of the four principal directions (left, right, top, bottom) as a function of the time before (negative values on the horizontal axis) or after cue onset (positive values) for the conditions in which either positions left or right from fixation were cued (top row) or in which the top or bottom positions were cued (bottom row; illustrated in the small insets). To compute the histograms, a moving-window analysis with a window of $100 \mathrm{~ms}$ and time-steps of $1 \mathrm{~ms}$ was used.

studies, see Martinez-Conde et al., 2009). In order to further investigate the possibility of incorrectly detected microsaccades, the minimum duration was increased to $8 \mathrm{~ms}$ and $12 \mathrm{~ms}$. If incorrect detections are restricted to uniquely monocular microsaccades, the observed rates and directions of monocular microsaccades are expected to be more strongly influenced by a stricter duration criterion than the rates and directions for binocular microsaccades. Figure 10 shows the microsaccade rates (as a function of time) and directions (in the second before cue onset) for the different minimum durations. Whereas microsaccade rates decrease with a higher minimum duration (i.e., fewer microsaccades are detected), the shape of the microsaccade signature (Figure 10, left) and the distribution of microsaccade directions (Figure 10, right) are relatively unaffected. Moreover, observed binocular and monocular microsaccade rates decrease by similar amounts following an increase in the minimum duration, providing further support that monocular microsaccades do not merely reflect noise in the eye movement recordings.

\section{Discussion}

The present study investigated the role of three factors on the direction of microsaccades. The direction of covert attention affected microsaccades directions in an interval between 200 and $450 \mathrm{~ms}$ after cue onset. The distribution of possible target locations had a small effect on the direction of microsaccades. In contrast, whether microsaccades occurred in both eyes simultaneously (binocular microsaccades) or were restricted to one eye only (monocular microsaccades) strongly influenced the distribution of microsaccade directions. 

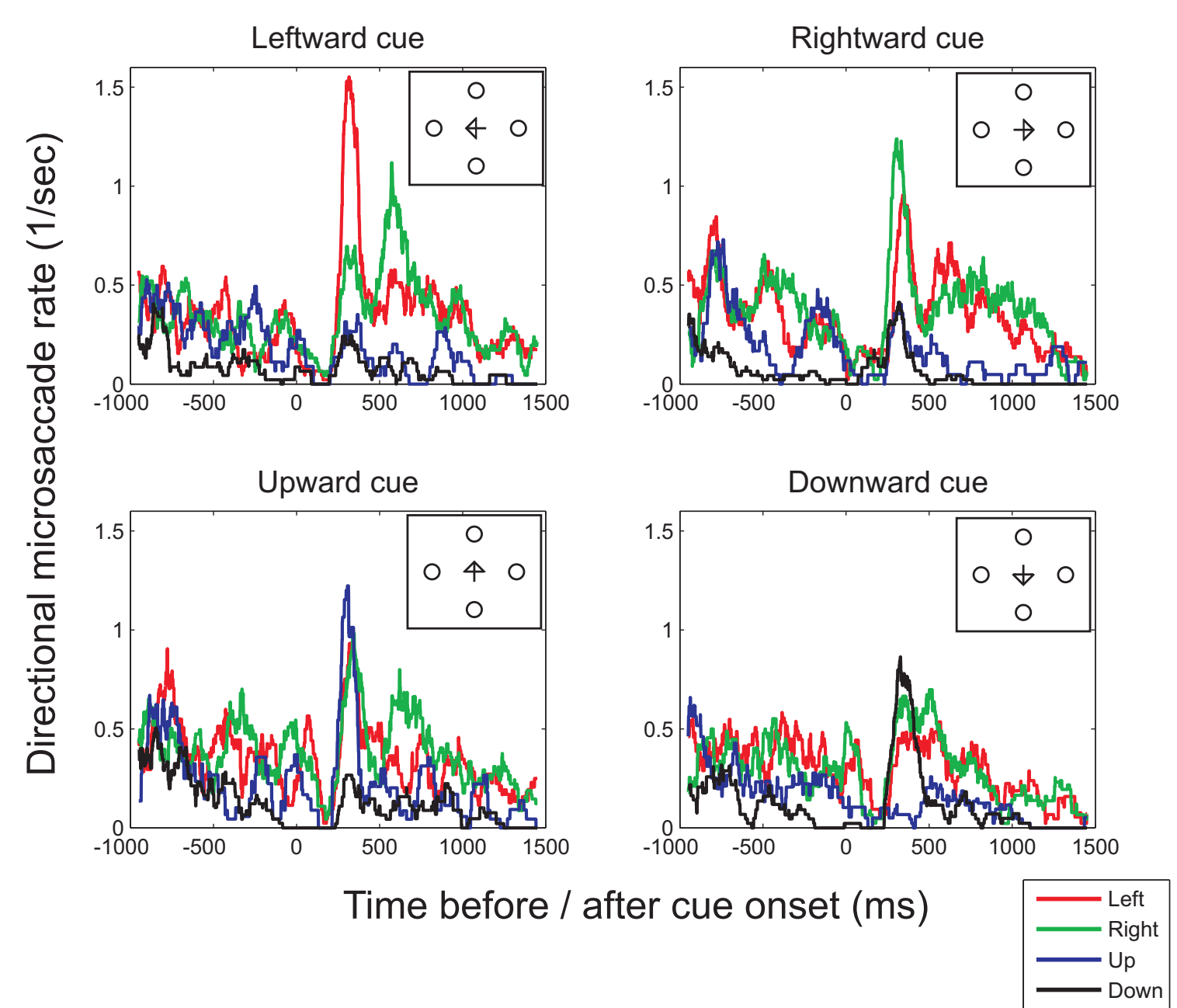

Figure 7. Binocular microsaccade rates in each of the four principal directions as a function of the time before (negative values on the horizontal axis) or after cue onset (positive values) in the condition in which the target could appear in one of the four locations (illustrated in the small insets). Histograms were computed using a moving window of $100 \mathrm{~ms}$ and time-steps of $1 \mathrm{~ms}$.

\section{Response times.}

An arrow cue, presented at the center of fixation, was used to direct participants' covert attention. In agreement with earlier observations (e.g., Gottlob, 2004), valid cues led to shorter saccadic latencies. These congruency effects suggest that the cues were effective in directing covert attention in the direction of the cue.

\section{Microsaccade frequency.}

Binocular microsaccades were found to be more frequent than monocular microsaccades. Despite this difference, a substantial proportion of microsaccades was found in uniquely one eye (39.9\% of the microsaccades were uniquely monocular events). Similar observations were made for the same eye tracking equipment and algorithm (Engbert \& Kliegl, 2003b; Kloke et al., 2009). The binocular microsaccades and the combined monocular and binocular microsaccades showed the characteristic microsaccade signature (Betta \& Turatto,
2006; Betta et al., 2007; Engbert \& Kliegl, 2003a; Galfano et al., 2004; Laubrock et al., 2005; Turatto et al., 2007) with a reduction of the microsaccade frequency after the onset of the cue followed by an increase above baseline (although the exact shape of the function appears to depend on the type of stimulus, Engbert \& Kliegl, 2003a; Laubrock et al., 2005; Rolfs, Kliegl, \& Engbert, 2008, and the algorithm used to produce the curve, with sharper peaks and a clearer increase above baseline for an unweighted average than for a filter based on neural firing rates, Engbert, 2006; Rolfs et al., 2008). This shows that in order to obtain the microsaccade signature, it is sufficient to record from only one eye. For uniquely monocular microsaccades, the modulation of the microsaccade rate was reduced (for the left eye) or virtually absent (for the right eye), suggesting that the two types of microsaccades (binocular and uniquely monocular) might have a different origin. Alternatively, they could be generated by the same mechanism, but might be affected differently by stimulus on- 
a)
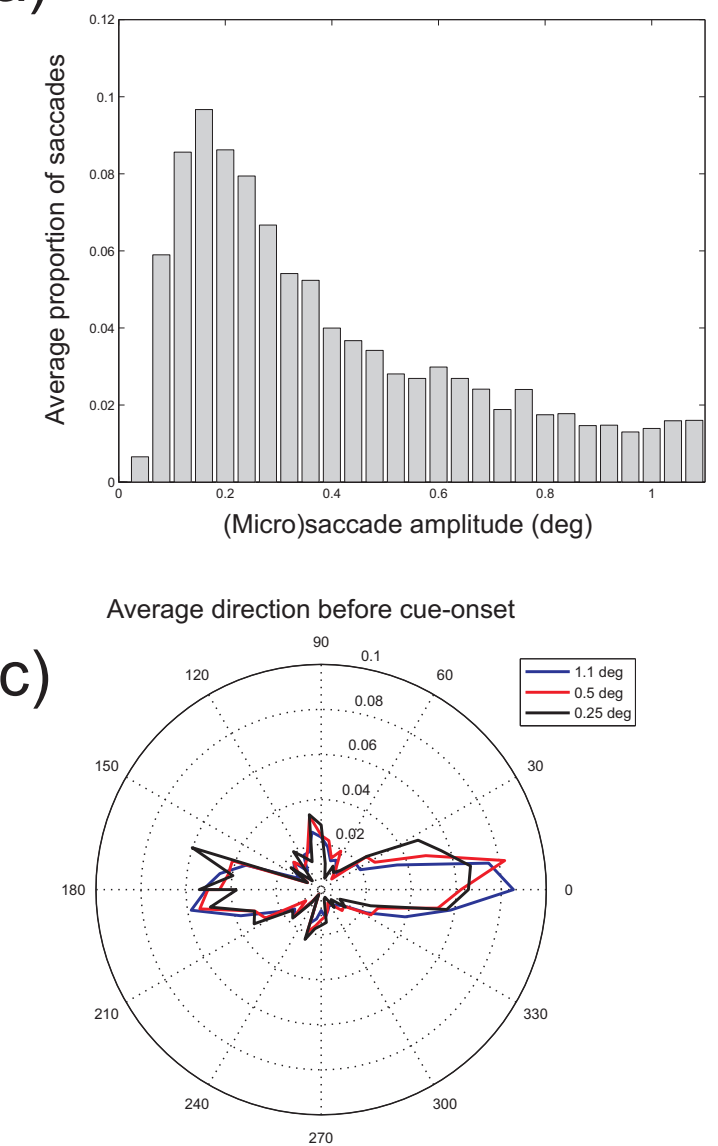

b)

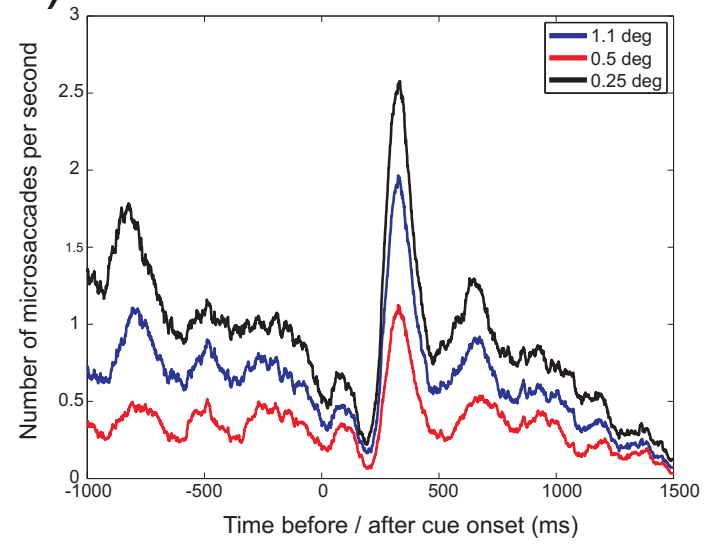

d)
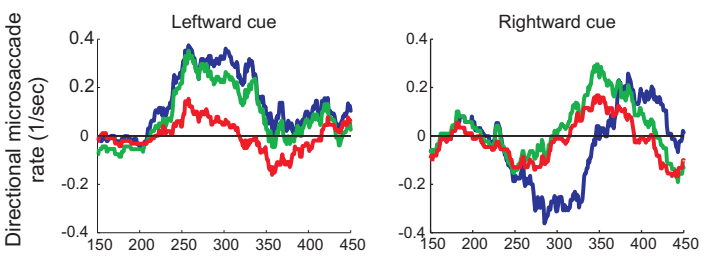

Time before/after cue onset (ms)

Figure 8. a) Histogram of the amplitude of binocular saccades detected during visual fixation. b) Microsaccade rate (horizontal cue condition) as a function of the time before (negative values on the horizontal axis) and after cue onset (positive values) for three microsaccade amplitude thresholds (1.1deg, $0.5 \mathrm{deg}, 0.25 \mathrm{deg})$. c) Histogram of microsaccade direction (horizontal cue condition) before cue onset for the three amplitude thresholds. d) Difference in the microsaccade leftward (+ve) and rightward (-ve) rates for leftward and rightward cues and for the three amplitude thresholds in the 150-450ms intveral after cue onset.

sets.

Large differences were found in the microsaccade rates across different participants (see also Engbert \& Kliegl, 2003a, 2003b). The same criteria were used for each participant and therefore it is not clear whether these differences reflect actual differences in microsaccade rates or whether the algorithm (Engbert \& Kliegl, 2003a; Engbert \& Mergenthaler, 2006) might be more sensitive at detecting microsaccades from the records of certain individuals (related to differences in the noiselevel of the recordings and calibration differences, or the properties of other fixational eye movements, such as slow drift; Mergenthaler \& Engbert, submitted). Related to this issue is the finding that, for our particular setup and calibration procedure, slightly higher microsaccade rates were found for the right eye than for the left eye in most participants. This could be an indication of differences in the measurement noise level for the two eyes, possibly related to differences in the two cameras or settings.
Similar microsaccades rates were found in the conditions with two possible target locations and the condition where the target could appear in four different locations, suggesting that increasing uncertainty about the target location does not change the frequency of microsaccades. When two possible target locations were used, larger numbers of microsaccades were found when vertical cues and place-holders were used, requiring participants to make vertical saccades, than for horizontal cues and place-holders. This higher rate for vertical cues was found across almost the entire interval, but specifically before cue onset. At this point, it is not clear what caused these higher rates and whether they would extend to different cueing situations, for example, using exogenous instead of endogenous cues.

\section{Microsaccade direction: attention.}

We started with the hypothesis that if attention strongly affects the direction of microsaccades, a large shift towards vertical microsaccades would be ex- 

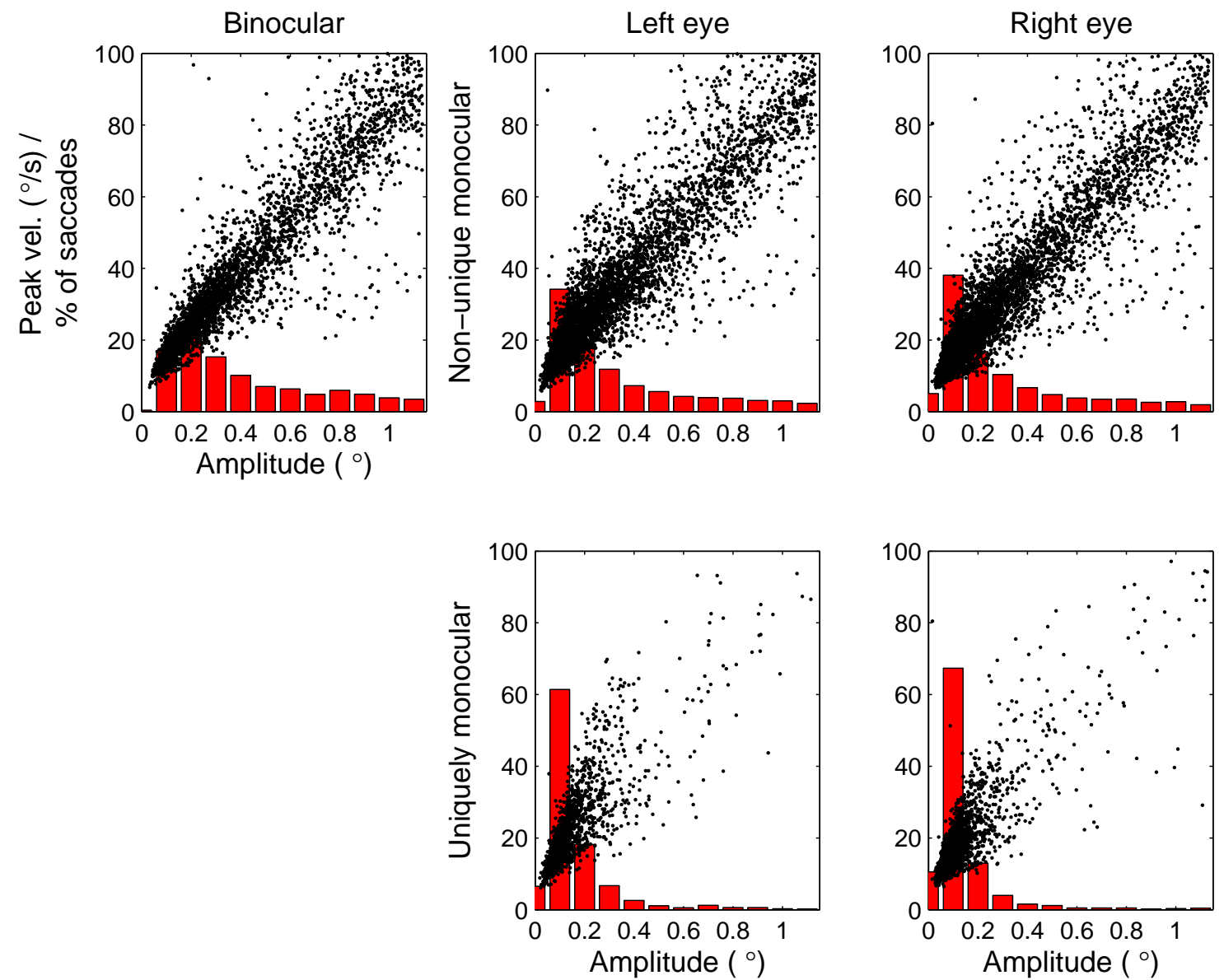

Figure 9. Scatterplots (black dots) plotting saccade amplitude (horizontal axis) against peak velocity (vertical axis) for the different types of saccades (binocular, non-unique monocular and uniquely monocular microsaccades, either in the left or right eye). Red bars in the background show histograms of saccade amplitudes.

pected if attention is cued in the vertical rather than in the horizontal direction. No such strong bias towards the vertical direction was observed. Instead, microsaccades, and in particular binocular microsaccades, remained mainly in the horizontal direction. By determining the time-course of microsaccade directions, however, a distinct bias of microsaccades in the direction of the cue was found in the interval between $250 \mathrm{~ms}$ to $400 \mathrm{~ms}$ after cue onset. Although it cannot be excluded that such biases were the result of microsaccades following the direction of the change in the fixation symbol rather than covert attention, there are two reasons why such an explanation is unlikely. First, it has been shown that color cues can also bias microsaccades in the presumed direction of covert attention (Abadi \& Gowen, 2004; Engbert \& Kliegl, 2003a; Laubrock et al., 2005), although biases tend to be stronger for arrow than for color cues (Engbert \& Kliegl, 2003a). Second, it has been shown that fixational eye movements are not influenced by the size or shape of the symbol at fixation (Murphy, Haddad, \& Steinman, 1974), suggesting that people can maintain fixa- tion even if the fixation symbol changes shape.

\section{Microsaccade direction: Possible target locations.}

Our data suggest that the locations in which targets could appear, plays a role, but that this factor has only a weak effect on the bias of microsaccades. When vertical targets were used, microsaccades tended to be slightly less often in the horizontal direction and slightly more often in the vertical direction compared to when horizontal targets were used. The effect of vertical targets appeared to be independent of whether horizontal targets were used as well. These effects were present before the onset of the arrow cue, which indicates that they were not the consequence of small saccades towards the arrow-head (Engbert \& Kliegl, 2003a).

The small effect of the possible target locations could have two possible sources. One is a bottom-up, stimulus driven mechanism by which the mere presence of the place-holders influences the bias of microsaccade directions. The other is a top-down influence, arising from expectations of where the target could appear. In- 
Journal of Eye Movement Research

$3(4): 1,1-20$
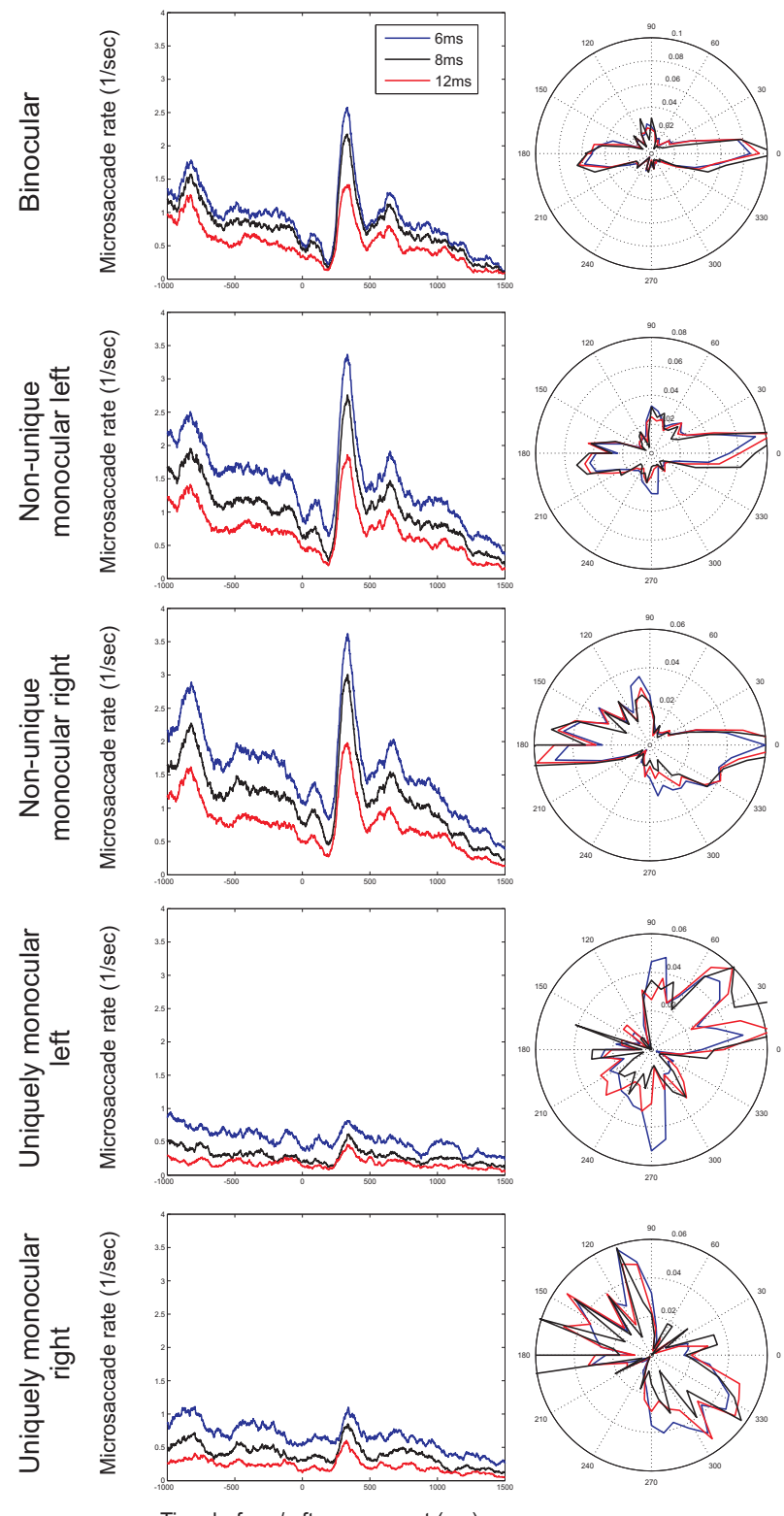

Figure 10. Microsaccade rates as a function of time before and after cue onset (left) and microsaccade directions before cue onset (right) for different minimum durations of microsaccades $(6 \mathrm{~ms}, 8 \mathrm{~ms}$, and $12 \mathrm{~ms}$; presented results restricted to horizontal cue trials).

dependent of which mechanism caused the present effects, the relatively weak modulation of microsaccade bias by the possible target locations indicates that this factor is not likely to explain why previous studies found microsaccades in many directions (Pastukhov \& Braun, 2010; Turatto et al., 2007).
Hermens, F. \& Walker, R. (2010)

What determines the direction of microsaccades?

\section{Microsaccade direction: Monocular and binocular microsaccades.}

Our data replicated earlier observations (Engbert \& Kliegl, 2003b; Kloke et al., 2009) showing that binocular microsaccades occur mainly in the horizontal direction, non-unique monocular microsaccades in both the horizontal and the vertical directions, and uniquely monocular microsaccades more often in the vertical direction. The combined horizontal and vertical bias of nonunique monocular microsaccades can be explained by realizing that the histogram for these microsaccades consists of the combined histograms of binocular and uniquely monocular microsaccades, which have a horizontal and a vertical bias respectively. After cue-onset, a similar pattern appear to be present, although the histograms for microsaccades after cue onset are less consistent. A possible source of this lack in consistency in the histograms after cue onset could be the smaller number of observations underlying each histogram. The average microsaccade rate was higher before cue onset (1.2 per second) than after cue onset ( 0.86 per second). This might have been compensated by the longer interval after cue onset (1.5 seconds versus 1 second before cue onset), were it not that for the histograms after cue onset the data were split across the different cue directions, and therefore each histogram uses approximately half or a quarter of the number of microsaccades of those available before cue onset.

Our data suggest that comparisons of results from studies using monocular recordings and those using a binocular criterion for microsaccades should be made with much care (Engbert \& Kliegl, 2003b; Kloke et al., 2009, current study). Earlier studies, using high precision eye trackers, reported microsaccades to be almost uniquely binocular events (Krauskopf, Cornsweet, \& Riggs, 1960; Riggs \& Niehl, 1960) (cited in Findlay, 2003), which has led some researchers to rely on recordings from one eye only for the detection of microsaccades (e.g., Findlay, 1974b). Our results suggest that such decisions, however, should be be taken lightly. Whether monocular microsaccades truly exist might remain a matter of debate, although our recordings with artificial 'dummy' pupils show that only few monocular microsaccades are detected for completely static 'eyes' (and false detections appeared to be eliminated completely by restricting the detection to eye movements that occurred in both eyes simultaneously, i.e., for 'binocular microsaccades'). The suggestion that the observed monocular microsaccades were not the result of false detections was supported by two additional observations. First, although monocular microsaccades showed a bias towards lower saccade amplitudes, they could be shown to follow the main sequence. Second, increasing the minimum duration of the saccade for detection affected binocular and monocular microsaccade rates in a similar way, did not eliminate the detection of uniquely monocular microsaccades and had little ef- 
Journal of Eye Movement Research

$3(4): 1,1-20$

fect on the distribution of both binocular and monocular microsaccade directions. Taken together, these results suggest that monocular microsaccades, if detected, should not be ignored. However, because they show different direction biases and they fail to show the typical microsaccade signature, we strongly recommend that they are analyzed separately from binocular microsaccades.

\section{Other factors that could determine microsaccade direction}

Clearly, there are many factors that could influence the direction of microsaccades and some of these are discussed below.

Analyzing horizontal and vertical components of eye movements. Several studies have relied on analyzing only the horizontal component of eye movements, because less accurate recordings could be obtained in the vertical direction (e.g., Hafed \& Clark, 2002). Our data suggest that this is a valid strategy if attention is cued in the horizontal direction only. To detect congruent biases of microsaccade direction for vertical cues, the vertical component needs to be measured and analyzed. In addition, data needs to be presented separately for the different cue directions, so that it can be evaluated whether microsaccade direction follows attention for all cue directions. Pooling the data across directions could obscure possible differential effects for different cue directions.

Exogenous and endogenous cueing. Differences have been found in the effects of exogenous and endogenous cues on microsaccade directions. Exogenous cues have been reported to induce a bias of microsaccades away from the direction of the cue, possible preceded by a bias towards the cue (Betta et al., 2007; Gowen, Abadi, Poliakoff, Hansen, \& Miall, 2007; Laubrock et al., 2005; Turatto et al., 2007). In contrast, endogenous cues typically result in a bias in the direction of the cue (present study and Engbert \& Kliegl, 2003a; Gowen et al., 2007; Laubrock et al., 2005). Such a bias towards the direction of endogenous cues has been found both for arrow and color cues. Interestingly, the interval at which the biases, if present, occur, compares to that reported for exogenous and endogenous cueing and, for exogenous cues, inhibition of return (Müller \& Rabbitt, 1989).

For endogenous cues, it is more difficult to construct a cue that will induce a clear shift of covert attention, without inducing small eye movements that could reflect refixations of the fixation symbol, possibly towards the center of gravity of the element presented at fixation. There are indications that the shape of the element at fixation does not influence fixation stability (Murphy et al., 1974), although the situation might be
Hermens, F. \& Walker, R. (2010)

What determines the direction of microsaccades?

different for stimuli that change at fixation. Future research could possibly rely on arrow symbols that are not presented at fixation, but symmetrically left and right of fixation (Hermens \& Walker, in press; Kuhn \& Benson, 2007), as well as on gaze cues, with the pupils of the eyes presented next to fixation (Deaner \& Platt, 2003).

Interval for analysis. For the selection of the interval across which microsaccades are pooled into one analysis, a trade-off has to be made between being sufficiently selective in order not to pool events from different episodes into one analysis and taking a large enough interval to have sufficient observations to perform a reliable analysis. The requirement not to take a too large interval seems to be particularly important when exogenous cues are considered, as for these cues there have been reports of two episodes of microsaccadic modulations with opposite biases (Laubrock et al., 2007). Pooling across such intervals of opposite microsaccade directions obviously results in the cancellation of any existing biases.

A related issue concerns the inclusion of microsaccades that occur in pairs (Hafed \& Clark, 2002). Although it has not yet been settled whether paired fixational eye movements should be classified as saccadic intrusions rather than microsaccades (Gowen et al., 2007), assume, for the current argument, that they fall into the class of microsaccades. In these pairs of small eye movements during fixation, a jump in one direction is often followed by a movement in the opposite direction (either saccadic or drift), bringing the eye back to its original position. If both microsaccades of such pairs are analyzed together, this could result an absence of a bias in the overall data, because the two directions of the eye movements that make up the pair cancel each other out. To avoid possible confound, some studies have therefore analyzed the direction of the first microsaccade in each pair separately (Hafed \& Clark, 2002) or investigated the direction of the first microsaccade in a particular interval (Deaner \& Platt, 2003; Laubrock et al., in press). A direct comparison of the microsaccades that occurred in pairs and those in isolation ('single-sided' microsaccades), however, demonstrated that both types of microsaccades show similar biases (Hafed \& Clark, 2002). Therefore, even if one would pool all microsaccades across a particular interval, including the second microsaccade of each pair, an overall direction bias should be found. Such a conclusion is consistent with the present data in which no reliable differences were found between the results of an analysis using all saccades within an interval after cue onset and an analysis of just the first microsaccade in that interval.

Decisions, for example, to separate microsaccades in pairs and single events, and to analyze only the first microsaccade in a particular interval, however, require 
Journal of Eye Movement Research

$3(4): 1,1-20$

some choices on how pairs are detected and on which interval is analyzed. These choices are not arbitrary. For example, to establish whether a microsaccade is part of a pair, one has to establish which microsaccade is the first of a pair and how much a second microsaccade can be delayed to still be considered to be part of a pair. Assume, for example, that three microsaccades occur in rapid succession. How should one decide which two are part of a pair and which one is a singlesided microsaccade? If one instead analyzes the first microsaccade within a particular interval, one should choose from which point in time after cue onset, such microsaccades need to be analyzed. Because of neural transmission times, it would probably best to avoid analyzing the first microsaccade within $\pm 100 \mathrm{~ms}$ after cue onset. In addition, the inclusion in the analysis of first microsaccades that occur well after the onset of the cue should be avoided. However, it is not clear how to set these boundaries on which first microsaccades to analyze and how and whether these criteria should vary across participants and stimulus conditions.

Eye tracking equipment. A large range of devices has been used to study microsaccades (see MartinezConde et al., 2009, for an overview). These range from custom-built systems (e.g., Findlay, 1974a) to widely available video-based eye trackers. It appears that the properties of the detected microsaccades are not completely independent of the system that is used for the recordings (Collewijn \& Kowler, 2008), although there is also a role for the algorithm and criteria used for microsaccade detection (such as criteria on the minimum duration and amplitude). To establish the individual contributions of the recording system and the detection algorithm, a direct comparison using different eye trackers would be required. To our knowledge, only one study performed such a comparison of two systems (Abadi \& Gowen, 2004). This study, however, focused on saccadic intrusions, which tend to be of relatively large amplitude, and therefore additional research would be required to determine whether these findings extend to fixational eye movements of smaller amplitudes.

Detection algorithm. Most recent studies of microsaccades have used the algorithm by Engbert and colleagues (Engbert \& Kliegl, 2003a; Engbert \& Mergenthaler, 2006), in which microsaccades are detected on the basis of a velocity criterion while taking into account the noise levels in the data. Additional requirements for a successful detection of a microsaccade often made are a minimum duration (e.g., 12ms), a temporal overlap of the detected saccades between the two eyes, and a maximum amplitude (e.g., 1deg) (see Martinez-Conde et al., 2009, for an overview). Alternative automated detection algorithms were developed by Martinez-Conde and colleagues (Martinez-Conde,
Hermens, F. \& Walker, R. (2010)

What determines the direction of microsaccades?

Macknik, \& Hubel, 2000) and Zanker and Walker (2004). At least one study made a comparison between the results using the procedure by Engbert and colleagues and that by Martinez-Conde et al., reporting no obvious differences (Otero-Millan, Troncoso, Macknik, Serrano-Pedraza, \& Martinez-Conde, 2008). In contrast, earlier studies of microsaccades appear to rely more often on detection by visual inspection, possibly resulting in fewer detected microsaccades, in particular when a more restricted amplitude criterion is used (Otero-Millan et al., 2008).

Our results suggest that the criterion for microsaccades to occur in both eyes simultaneously is an important factor for video-based eye trackers, especially if microsaccade direction biases rather than frequencies are studied, and it is therefore important to state if the recording was monocular or binocular. In contrast, the exact amplitude criterion and the minimum duration of the saccade appears to be of less importance. Although fewer microsaccades are detected with a stricter amplitude and duration criteria, the main features of the data, such as the microsaccade signature and the horizontal bias for binocular microsaccades appear to be maintained (see also Poletti \& Rucci, 2010). Reducing the amplitude criterion resulted in a smaller bias of microsaccades in the direction of attention, although an earlier study (Hafed \& Clark, 2002) has suggested that also this property of the data is maintained with a more stringent amplitude criterion.

The 1 degree amplitude criterion typically applied (Martinez-Conde et al., 2009) might cause the inclusion of saccadic intrusions into the pool of data (in addition to the microsaccades, which are usually believed to be of smaller amplitude; Collewijn \& Kowler, 2008; Gowen et al., 2007). Inspection of the amplitude histograms suggests that if such a possible confound between microsaccades and saccadic intrusions occurs, the contribution of saccadic intrusions is relatively modest, because larger amplitude fixational saccadic eye movements ( $>0.5 \mathrm{deg}$ ) appear to be relatively infrequent (Engbert, 2006; Otero-Millan et al., 2008; Poletti \& Rucci, 2010, present data). For statistics of microsaccade amplitude, however, the contribution of saccadic intrusions might best be avoided by using median rather than mean data.

The range of microsaccade amplitudes could also be influenced by the fixation requirements in the experiment. Experiments that required fixation within a particular region (e.g., <1deg) around a central position on the display (Horwitz \& Albright, 2003; Tse et al., 2004) might have resulted in a smaller range of amplitudes. Such a conclusion would be in line with the finding that microsaccades are less frequent when no fixation marker is used (Hermens, Walker, \& Zanker, unpublished results and Poletti \& Rucci, 2010), possibly because of an increase in slow drift (Nachmias, 1959, 1961) (as cited in Kowler, 1990). 
Journal of Eye Movement Research

$3(4): 1,1-20$

Statistical analysis. Histograms of microsaccade frequency or directions need to be compared statistically across conditions in order to determine whether there are biases in a particular direction dependent on the stimulus condition. Such comparisons of the histograms is not entirely without issues. For example, when considering microsaccade directions, microsaccades are found to biased in the horizontal direction, which means that most cells of the histogram contain zero or close to zero observations, whereas cells with directions close to the horizontal contain large numbers. If one would decide to compare the values across all cells, this would bias the comparison to the many cells with close to zero observations. A comparison between the cells with many observations only would require a decision on which cells to consider. Such comparisons also depend on the number of bins used to construct the histograms. Possibly, procedures using surrogate data (Engbert \& Mergenthaler, 2006) provide better evaluations of the statistical significance of the results, although it might not always be clear whether all assumptions underlying such procedures are satisfied (e.g., concerning within and between subject variance in the data).

The issue concerning the size of the interval across which rates or direction biases are compared, also applies to statistical testing. Ideally, rates and biases should be compared across a smallest possible interval (e.g., $2 \mathrm{~ms}$, the sampling interval) for each moment before and after cue onset. However, small intervals have the disadvantage of many multiple comparisons, which increases the chance of a type I statistical error if no correction is made, or lead to very conservative tests if corrections are applied. The method that we used, in which microsaccade rates were averaged across an interval selected on the basis of the data plots has its problems as well. By taking a larger interval, multiple effects that occur at different delays from cue onset could have been averaged, with the consequence that only the summed effect is examined. Furthermore, biases could have arisen by selecting the intervals for testing on the basis of the data. A preferred method would be to select an interval on the basis of a random subset of the participants, and to test the significance of the observed difference on the remaining participants (cross-validation), which, however, would require testing a large number of participants than presently used.

\section{Horizontal binocular microsaccades}

Our results are consistent with others showing that binocular microsaccades are biased in the horizontal direction. Engbert (2006) speculated that this bias might be the consequence of microsaccades being involuntary. Because of the restrictions on how the muscles rotate the eye, this would lead to eye movements in the four principal directions, avoiding oblique orientations. Alternatively, microsaccades, serving as cor-
Hermens, F. \& Walker, R. (2010)

What determines the direction of microsaccades?

rections for reductions of binocular disparity following disconjugate slow drifts, could be biased towards the horizontal, simply because of position of the eyes in the head. Another possibility would be that (micro)saccades are biased towards the horizontal, because of the distribution of visual elements in the display (Hansen \& Essock, 2004), which would be supported by our observation that the position of the placeholders affected the distribution of microsaccade directions (although only weakly).

\section{Conclusion}

Large differences between monocular and binocular microsaccade directions were found. Covert attention also influenced microsaccade directions, but only during a short interval after cue onset. The distribution of possible target locations only weakly affected microsaccade directions.

Binocularly and monocularly recorded microsaccade rates showed similar patterns of results (as long as microsaccades were not restricted to uniquely one eye), suggesting that microsaccade frequencies can be compared across experiments. Such direct comparisons, however, cannot always be made for microsaccade directions, where it is important to take into account whether eye movements were recorded in one or in two eyes simultaneously. Our findings suggests that it is important to consider the exact protocol used to measure microsaccades when comparing results across different studies.

\section{References}

Abadi, R. V., \& Gowen, E. (2004). Characteristics of saccadic intrusions. Vision Research, 44, 2675-2690.

Betta, E., Galfano, G., \& Turatto, M. (2007). Microsaccadic response during inhibition of return in a targettarget paradigm. Vision Research, 47(3), 428-436.

Betta, E., \& Turatto, M. (2006). Are you ready? I can tell by looking at your microsaccades. Neuroreport(17), 10011004.

Collewijn, H., \& Kowler, E. (2008). The significance of microsaccades for vision and oculomotor control. Journal of Vision, 8, 1-21.

Deaner, R. O., \& Platt, M. L. (2003). Reflexive social attention in monkeys and humans. Current Biology, 13, 1609-1613.

Engbert, R. (2006). Microsaccades: A microcosm for research on oculomotor control, attention, and visual perception. Progress in Brain Research, 154, 177-192.

Engbert, R., \& Kliegl, R. (2003a). Microsaccades uncover the orientation of covert attention. Vision Research, 43(9), 10351045.

Engbert, R., \& Kliegl, R. (2003b). Binocular coordination in microsaccades. In J. Hyönä, R. Radach, \& H. Deubel (Eds.), The mind's eyes: Cognitive and applied aspects of eye movements (p. 103-117). Oxford: Elsevier.

Engbert, R., \& Mergenthaler, K. (2006). Microsaccades are triggered by low retinal image slip. Procedings of the $\mathrm{Na}$ tional Academy of Sciences U.S.A., 103, 7192-7197. 
Journal of Eye Movement Research

$3(4): 1,1-20$

Findlay, J. M. (1974a). A simple apparatus for recording microsaccades during visual fixation. Quarterly Journal of Experimental Psychology, 26, 167-170.

Findlay, J. M. (1974b). Direction perception and human fixation eye movements. Vision Research, 14, 703-711.

Findlay, J. M. (2003). Eye movements and visual information processing. In J. Hyönä, R. Radach, \& H. Deubel (Eds.), The mind's eyes: Cognitive and applied aspects of eye movements (p. 143-155). Oxford: Elsevier.

Galfano, G., Betta, E., \& Turatto, M. (2004). Inhibition of return in microsaccades. Experimental Brain Research, 159(3), 400-404.

Gottlob, L. R. (2004). Location cuing and response time distributions in visual attention. Perception $\mathcal{E}$ Psychophysics, $66,1293-1302$.

Gowen, E., Abadi, R. V., Poliakoff, E., Hansen, P. C., \& Miall, R. C. (2007). Modulation of saccadic intrusions by exogenous and endogenous attention. Brain Research, 1141, 154-167.

Hafed, Z. M., \& Clark, J. J. (2002). Microsaccades as an overt measure of covert attention shifts. Vision Research, 42, 2533-2545.

Hafed, Z. M., Goffart, L., \& Krauzlis, R. J. (2009). A neural mechanism for microsaccade generation in the primate superior colliculus. Science, 323, 940-943.

Hansen, B. C., \& Essock, E. A. (2004). A horizontal bias in human visual processing of orientation and its correspondence to the structural components of natural scenes. Journal of Vision, 4(12:5), 1044-1060.

Hermens, F., \& Walker, R. (in press). Gaze and arrow distractors influence saccade trajectories similarly. Quarterly Journal of Experimental Psychology.

Hermens, F., Zanker, J. M., \& Walker, R. (2010). Microsaccades and preparatory set: A comparison between delayed and immediate, exogenous and endogenous proand anti-saccades. Experimental Brain Research, 201(3), 489498.

Horowitz, T. S., Fine, E. M., Fencsik, D. E., Yurgenson, S., \& Wolfe, J. M. (2007). Fixational eye movements are not an index of covert attention. Psychological Science, 18, 356-363.

Horwitz, G. D., \& Albright, T. D. (2003). Short-latency fixational saccades induced by luminance increments. Journal of Neurophysiology, 90, 1333-1339.

Kloke, W. B., Jaschinski, W., \& Jainta, S. (2009). Microsaccades under monocular viewing conditions. Journal of Eye Movement Research, 3(1), 1-7.

Kowler, E. (1990). The role of visual and cognitive processes in the control of eye movement. In E. Kowler (Ed.), Eye movements and their role in visual and cognitive processes. Elsevier, Amsterdam.

Kowler, E., \& Steinman, R. M. (1980). Small saccades serve no useful purpose: reply to a letter by R. W. Ditchburn. Vision Research, 20, 273-276.

Krauskopf, J., Cornsweet, T. N., \& Riggs, L. A. (1960). Analysis of eye movements during monocular and binocular fixation. Journal of the Optical Society of America, 50, 572578.

Kuhn, G., \& Benson, V. (2007). The influence of eye-gaze and arrow pointing distractor cues on voluntary eye movements. Perception \& Psychophysics, 69, 966-971.
Hermens, F. \& Walker, R. (2010)

What determines the direction of microsaccades?

Laubrock, J., Engbert, R., \& Kliegl, R. (2005). Microsaccade dynamics during covert attention. Vision Research, 45(6), 721-730.

Laubrock, J., Engbert, R., Rolfs, M., \& Kliegl, R. (2007). Microsaccades are an index of covert attention: commentary on Horowitz, Fine, Fencsik, Yurgenson, and Wolfe (2007). Psychological Science, 18(4), 364-366.

Laubrock, J., Kliegl, R., Rolfs, M., \& Engbert, R. (in press). When do microsaccades follow attention? Attention, Perception, E Psychophysics.

Martinez-Conde, S. (2006). Fixational eye movements in normal and pathological vision. Progress in Brain Research, 154, 151-176.

Martinez-Conde, S., Macknik, S. L., \& Hubel, D. H. (2000). Microsaccadic eye movements and firing of single cells in the striate cortex of macaque monkeys. Nature Neuroscience, 3, 251-258.

Martinez-Conde, S., Macknik, S. L., Troncoso, X. G., \& Dyar, T. A. (2006). Microsaccades counteract visual fading during fixation. Neuron, 49(2), 297-305.

Martinez-Conde, S., Macknik, S. L., Troncoso, X. G., \& Hubel, D. H. (2009). Microsaccades: a neurophysiological analysis. Trends in Neurosciences.

Mergenthaler, K., \& Engbert, R. (submitted). Microsaccade detection: Statistical testing using surrogate data and effects of inter-individual differences and variations in luminance.

Müller, H. J., \& Rabbitt, P. M. (1989). Reflexive and voluntary orienting of visual attention: time course of activation and resistance to interruption. Journal of Experimental Psychology: Humand Perception \& Performance, 15, 315-330.

Murphy, B. J., Haddad, G. M., \& Steinman, R. M. (1974). Simple forms and fluctuations of the line of sight: Implications for motor theories of form processing. Perception $\mathcal{E}$ Psychophysics, 16, 557-563.

Nachmias, J. (1959). Two-dimensional motion of the retinal image during monocular fixation. Journal of the Optical Society of America, 49, 901-908.

Nachmias, J. (1961). Determiners of the drift of the eye during monocular fixation. Journal of the Optical Society of America, 51, 761-766.

Otero-Millan, J., Troncoso, X. G., Macknik, S. L., SerranoPedraza, I., \& Martinez-Conde, S. (2008). Saccades and microsaccades during visual fixation, exploration, and search: foundations for a common saccadic generator. Journal of Vision, 8, 1-18.

Pastukhov, A., \& Braun, J. (2010). Rare but precious: microsaccades are highly informative about attentional allocation. Vision Research.

Poletti, M., \& Rucci, M. (2010). Eye movements under various conditions of image fading. Journal of Vision, 10(3:6), 1-18.

Posner, M. I. (1980). Orienting of attention. Quarterly Journal of Experimental Psychology, 32, 3-25.

Riggs, L. A., \& Niehl, F. W. (1960). Eye movements recorded during convergence and divergence. Journal of the Optical Society of America, 50, 913-920.

Rolfs, M. (2009). Microsaccades: Small steps on a long way. Vision Research.

Rolfs, M., Engbert, R., \& Kliegl, R. (2004). Microsaccade orientation supports attentional enhancement opposite a 
peripheral cue: commentary on Tse, Sheinberg, and Logothetis (2003). Psychological Science, 15(10), 705-707.

Rolfs, M., Engbert, R., \& Kliegl, R. (2005). Crossmodal coupling of oculomotor control and spatial attention in vision and audition. Experimental Brain Research, 166, 427-439.

Rolfs, M., Kliegl, R., \& Engbert, R. (2008). Towards a model of microsaccade generation: The case of microsaccadic inhibition. Journal of Vision.

Rolfs, M., Laubrock, J., \& Kliegl, R. (2006). Shortening and prolongation of saccade latencies following microsaccades. Experimental Brain Research, 169, 369-376.

Rucci, M., Iovin, R., Poletti, M., \& Santini, F. (2007). Miniature eye movements enhance fine spatial detail. Nature, 447(7146), 851-854.

Steinman, R. M., Haddad, G. M., Skavenski, A. A., \& Wyman, D. (1973). Miniature eye movement. Science, 181, 810-819.

Tse, P. U., Sheinberg, D. L., \& Logothetis, N. K. (2002). Fixational eye movements are not affected by abrupt onsets that capture attention. Vision Research, 42(13), 1663-1669.

Tse, P. U., Sheinberg, D. S., \& Logothetis, N. K. (2004). The distribution of microsaccade directions need not reveal the location of attention. Psychological Science, 15, 708-710.

Turatto, M., Valsecchi, M., Tame, L., \& Betta, E. (2007). Microsaccades distinguish between global and local visual processing. Neuroreport, 18(10), 1015-1018.

Valsecchi, M., Betta, E., \& Turatto, M. (2007). Visual oddballs induce prolonged microsaccadic inhibition. Experimental Brain Research, 177(2), 196-208.

Zanker, J. M., \& Walker, R. (2004). A new look at Op art: towards a simple explanation of illusory motion. Naturwissenschaften, 91, 149-156.

Zuber, B. L., Stark, L., \& Cook, G. (1965). Microsaccades and the velocity-amplitude relationship for saccadic eye movements. Science, 150(3702), 1459-1460. 